TRANSACTIONS OF THE

AMERICAN MATHEMATICAL SOCIETY

Volume 353, Number 2, Pages 655-692

S 0002-9947(00)02663-5

Article electronically published on September 27, 2000

\title{
UNIQUENESS OF SOLUTION TO A FREE BOUNDARY PROBLEM FROM COMBUSTION
}

\author{
C. LEDERMAN, J. L. VÁZQUEZ, AND N. WOLANSKI
}

\begin{abstract}
We investigate the uniqueness and agreement between different kinds of solutions for a free boundary problem in heat propagation that in classical terms is formulated as follows: to find a continuous function $u(x, t) \geq$ 0 , defined in a domain $\mathcal{D} \subset \mathbb{R}^{N} \times(0, T)$ and such that
\end{abstract}

$$
\Delta u+\sum a_{i} u_{x_{i}}-u_{t}=0 \text { in } \mathcal{D} \cap\{u>0\} .
$$

We also assume that the interior boundary of the positivity set, $\mathcal{D} \cap \partial\{u>0\}$, so-called free boundary, is a regular hypersurface on which the following conditions are satisfied:

$$
u=0, \quad-\partial u / \partial \nu=C .
$$

Here $\nu$ denotes outward unit spatial normal to the free boundary. In addition, initial data are specified, as well as either Dirichlet or Neumann data on the parabolic boundary of $\mathcal{D}$. This problem arises in combustion theory as a limit situation in the propagation of premixed flames (high activation energy limit).

The problem admits classical solutions only for good data and for small times. Several generalized concepts of solution have been proposed, among them the concepts of limit solution and viscosity solution. We investigate conditions under which the three concepts agree and produce a unique solution.

\section{INTRODUCTION}

In this paper we deal with a free boundary problem in heat propagation which in its classical form can be formulated as follows: given a domain $\mathcal{D} \subset \mathbb{R}^{N} \times(0, T)$, the problem consists in finding a nonnegative function $u(x, t)$, defined and continuous in $\mathcal{D}$, which satisfies the equation

$$
\Delta u+\sum a_{i} u_{x_{i}}-u_{t}=0
$$

in the positivity set $\mathcal{D} \cap\{u>0\}$. We also assume that the interior boundary of the positivity set, $\Gamma=\mathcal{D} \cap \partial\{u>0\}$, so-called free boundary, is a regular hypersurface

Received by the editors April 2, 1999.

1991 Mathematics Subject Classification. Primary 35K05, 35K60, 80A25.

Key words and phrases. Free-boundary problem, combustion, heat equation, uniqueness, classical solution, viscosity solution, limit solution.

The first and third authors were partially supported by UBA grants EX071, TX47 and grant BID802/OC-AR PICT 03-00000-00137. They are members of CONICET (Consejo Nacional de Investigaciones Científicas y Técnicas of Argentina).

The second author was partially supported by DGICYT Project PB94-0153 and HCM contract FMRX-CT98-0201. 
on which the following conditions are satisfied

$$
u=0, \quad-\frac{\partial u}{\partial \nu}=\sqrt{2 M} .
$$

Here $M$ is a positive constant, and $\nu$ denotes outward unit spatial normal to the free boundary $\Gamma$. In addition, initial and boundary conditions have to be prescribed on the parabolic boundary of $\mathcal{D}$. Thus, if the domain is a space-time cylinder, $\mathcal{D}=\Omega \times(0, T)$, we prescribe initial data at $t=0$

$$
u(x, 0)=u_{0}(x) \quad \text { for } x \in \bar{\Omega},
$$

as well as boundary conditions of Dirichlet or Neumann type on the lateral boundary, $\partial \Omega \times(0, T)$. We will refer to this free boundary problem as problem $P$. Let us recall that classical solutions to problem $P$ in one space dimension are relatively easy to construct; cf. [Ve]. The problem is much more difficult in several space dimensions; cf. [M], $\mathrm{AG}$. In general, classical solutions exist only locally in time, since singularities can arise in finite time. Classical solutions are constructed in GHV] under the assumption of radial symmetry.

Problem $P$ arises in several contexts and is currently the object of active investigation; cf. the survey paper $\nabla$. The most important motivation to date has come from combustion theory, where it appears as a limit situation in the description of the propagation of premixed equi-diffusional deflagration flames, which after convenient simplifications are reduced to solving the equation

$$
\Delta u^{\varepsilon}+\sum a_{i} u_{x_{i}}^{\varepsilon}-u_{t}^{\varepsilon}=\beta_{\varepsilon}\left(u^{\varepsilon}\right),
$$

for the variable $u^{\varepsilon}(x, t)=T_{f}-T(x, t)$, with $T$ the temperature of the reactive mixture and $T_{f}$ the flame temperature, so that $T \leq T_{f}$ and $u^{\varepsilon} \geq 0$. The function $\beta_{\varepsilon}(u)$ represents the exothermic chemical reaction and has accordingly a number of properties: it is a nonnegative and Lipschitz continuous function which is positive in an interval $\left(0, \theta_{\varepsilon}\right)$ near $u=0$ and vanishes otherwise (i.e., reaction occurs only in the range $T_{f}-\theta_{\varepsilon}<T<T_{f}$ ). The parameter $\varepsilon>0$ is essentially the inverse of the activation energy of the chemical reaction and plays an important role in the analysis. Finally, the integral $\int \beta_{\varepsilon}(u) d u=M$ is fixed. The vector $\left(a_{1}, \cdots, a_{N}\right)$ represents the transport velocity of the reactive mixture, which is sometimes taken to be zero in the quoted literature. For further details on the model see $[\mathrm{BuL}],[\mathrm{W}]$, $\mathrm{BL}$.

The important point in order to establish a connection of the two problems $P$ and $P_{\varepsilon}$ is that in the latter $\varepsilon$ is in many cases a very small parameter so that in the limit $\varepsilon \rightarrow 0$ (so-called limit of high activation energy) the support of the function $\beta_{\varepsilon}$ concentrates at $u=0$. The relevant limit happens when we let $\varepsilon \rightarrow 0$, and consequently $\theta_{\varepsilon} \rightarrow 0$, while keeping a constant integral $M$. The function $\beta_{\varepsilon}$ tends then to a Dirac delta, $M \delta(u)$. In this way the reaction zone where $\beta_{\varepsilon}$ acts is reduced to a surface, the flame front, and the free boundary problem arises. The fact that $M>0$ ensures that a nontrivial combustion process takes place so that a non-empty free boundary actually appears.

The study of the limit $P_{\varepsilon} \rightarrow P$ as $\varepsilon \rightarrow 0$ was proposed in the 1930's by Zeldovich and Frank-Kamenetski $[\mathrm{ZF}]$ and has been much discussed in the combustion literature. Although the convergence of the most relevant propagation modes, i.e., the traveling waves was already discussed by Zeldovich and Frank-Kamenetski, and has made enormous progress, a rigorous mathematical investigation of the convergence 
of general solutions is still in progress. Berestycki and his collaborators have rigorously studied the convergence problem for traveling waves and, more generally in the elliptic stationary case; cf. [BCN] and its references. See also [LW]. The study of the limit in the general evolution case for the heat operator has been performed in [CV] and extended in [C1], C2], CLW1 and CLW2 to the two-phase case, where no sign restriction on $u$ is made.

In any case, the validity of the free boundary model for general curved geometries is still under debate with many open problems waiting to be settled. Various concepts of generalized solution have been introduced in the literature in order to justify the limit process and to obtain solutions for general data. Thus, when we perform the approximation process $P_{\varepsilon}$ and pass to the limit $\varepsilon \rightarrow 0$, this gives rise to a kind of solutions to problem $P$, called limit solutions. In $[\mathrm{CV}]$ a concept of weak solution is introduced to clarify the nature of the limit solutions. On the other hand, the concept of viscosity solution for problem $P$ was introduced in [CLW2] and [LW] with the same purpose and the two-phase version of this problem is studied.

Let us recall that there exist other applications, approaches and solution concepts for which we refer to $[\nabla]$. For the case of the heat operator, $[\mathrm{HH}$ discusses the existence of generalized solutions to $P$ based on an elliptic-parabolic formulation in one space dimension, and such an approach is extended in GHV] to several dimensions under conditions of radial symmetry. Such solutions are shown to be classical until a singularity forms. The singularity is then classified.

As a precedent to this work we can quote the convergence results for traveling waves, starting from [BNS]. In [GHV] the classical solutions obtained by the ellipticparabolic approach are shown to coincide with the limit solutions of [CV]. Strong conditions are imposed, in particular radial symmetry in space. See [G] and [BoG] for recent work.

\section{MAin RESUlt AND OUtLine of THE PAPER}

It is the purpose of this work to contribute to the questions of unique characterization of the solution of the free-boundary problem $P$ and the consistency of the different solution concepts. Our results can be summarized as saying that, under suitable assumptions on the domain, the reaction function $\beta_{\varepsilon}$ and on the initial and boundary data, if a classical solution of problem $P$ exists in a certain time interval, then it is at the same time the unique classical solution, the unique limit solution and also the unique viscosity solution in that time interval.

For definiteness we take as spatial domain a cylinder of the form $\Omega=\mathbb{R} \times \Sigma$ with $\Sigma \subset \mathbb{R}^{N-1}$ a smooth domain, or a semi-cylinder, and we put homogeneous Neumann conditions on the lateral boundary $\mathbb{R} \times \partial \Sigma$. This is a usual choice in combustion problems. Meirmanov [M] constructs classical solutions in such domains in two dimensions (with periodic lateral conditions, however). It is worth recalling in this context that problem $P$ is not globally well-posed for general geometries where the solutions do develop singularities; see examples and discussion in $\mathrm{V}$. We require monotonicity of the initial data in the direction of the cylinder axis. On the contrary, we make no requirement of monotonicity of the solution in time. In the family of problems $P_{\varepsilon}$ we assume that the functions $\beta_{\varepsilon}$ are defined by scaling of a single function $\beta: \mathbb{R} \rightarrow \mathbb{R}$ satisfying:

(i) $\beta$ is a Lipschitz continuous function,

(ii) $\beta>0$ in $(0,1)$ and $\beta \equiv 0$ otherwise, 
(iii) $\int \beta(s) d s=M$.

We then define

$$
\beta_{\varepsilon}(s)=\frac{1}{\varepsilon} \beta\left(\frac{s}{\varepsilon}\right) .
$$

The coefficients $a_{i}$ in the operator are assumed to be independent of $x_{1}$, the direction of the cylinder axis, and belong to $C^{\alpha, \frac{\alpha}{2}}(\bar{\Sigma} \times[0, T])$.

Our result shows in particular that there is a unique limit solution independently of the choice of the function $\beta$. Moreover, we actually prove that the limit exists for any approximation of the initial datum.

An outline of the contents is as follows. In Section 2 we give precise definitions of the classical and viscosity solutions and prove a first consistency result (Propositions 2.1 and 2.2). In Section 3 we prove that, under certain assumptions on the domain and on the initial datum, a classical solution to problem $P$ is the unique classical solution and also the unique viscosity solution (Theorems 3.1 and 3.2 and Corollary 3.1). In Section 4 we prove some auxiliary results. In Section 5 we prove that a classical subsolution to problem $P$ is the uniform limit of a family of subsolutions to problem $P_{\varepsilon}$ and we prove the analogous result for supersolutions. Finally, in Section 6 we show that, under similar assumptions as in Section 3, a classical solution to problem $P$ is the uniform limit of any family of solutions to problem $P_{\varepsilon}$ (Theorems 6.1 and 6.2). We include an Appendix at the end of the paper with a result on parabolic semilinear mixed problems in noncylindrical space-time domains, that is used throughout the paper.

Notation. Throughout the paper $N$ will denote the spatial dimension, $\Sigma \subset \mathbb{R}^{N-1}$ a bounded $C^{3}$ domain with unit exterior normal $\eta^{\prime}$ and $\eta=\left(0, \eta^{\prime}\right)$ will denote the unit exterior normal to $\mathbb{R} \times \Sigma$. In addition, the following notation will be used:

For any $x_{0} \in \mathbb{R}^{N}, t_{0} \in \mathbb{R}$ and $\tau>0, B_{\tau}\left(x_{0}\right):=\left\{x \in \mathbb{R}^{N} /\left|x-x_{0}\right|<\tau\right\}$ and $B_{\tau}\left(x_{0}, t_{0}\right):=\left\{(x, t) \in \mathbb{R}^{N+1} /\left|x-x_{0}\right|^{2}+\left|t-t_{0}\right|^{2}<\tau^{2}\right\}$.

When necessary, we will denote points in $\mathbb{R}^{N}$ by $x=\left(x_{1}, x^{\prime}\right)$, with $x^{\prime} \in \mathbb{R}^{N-1}$. Given a function $v$, we will denote $v^{+}=\max (v, 0)$.

The symbols $\Delta$ and $\nabla$ will denote the corresponding operators in the space variables; the symbol $\partial_{p}$ applied to a domain will denote parabolic boundary.

Let us define the Hölder spaces we are going to use. Let $m \geq 0$ be an integer and $0<\alpha<1$. For a space-time cylinder $Q=\Omega \times(0, T) \subset \mathbb{R}^{N+1}, C^{m+\alpha, \frac{m+\alpha}{2}}(Q)$ is the parabolic Hölder space denoted by $H^{m+\alpha, \frac{m+\alpha}{2}}(Q)$ in [LSU]. If $\mathcal{D} \subset \mathbb{R}^{N+1}$ is a general domain, then $C^{m+\alpha, \frac{m+\alpha}{2}}(\mathcal{D})$ will denote the space of functions in $C^{m+\alpha, \frac{m+\alpha}{2}}(Q)$ for every space-time cylinder $Q \subset \mathcal{D}$. If $\mathcal{D}$ is bounded, we will say that $u \in C^{m+\alpha, \frac{m+\alpha}{2}}(\overline{\mathcal{D}})$ if there exist a domain $\mathcal{D}^{\prime}$ with $\overline{\mathcal{D}} \subset \mathcal{D}^{\prime}$ and a function $u^{\prime} \in C^{m+\alpha, \frac{m+\alpha}{2}}\left(\mathcal{D}^{\prime}\right)$ such that $u=u^{\prime}$ in $\overline{\mathcal{D}}$. If $\mathcal{D}$ is unbounded, we will say that $u \in C^{m+\alpha, \frac{m+\alpha}{2}}(\overline{\mathcal{D}})$ if $u \in C^{m+\alpha, \frac{m+\alpha}{2}}\left(\overline{\mathcal{D}^{\prime}}\right)$ for every bounded domain $\mathcal{D}^{\prime} \subset \mathcal{D}$. The space $C^{1}(\overline{\mathcal{D}})$ is defined in an analogous way.

In addition, $M$ will denote a positive constant that will remain fixed throughout the paper.

Given a domain $\mathcal{D} \subset \mathbb{R}^{N+1}$, we will write

$$
\mathcal{L} u:=\Delta u+\sum a_{i} u_{x_{i}}-u_{t}, \quad a_{i} \in L^{\infty}(\mathcal{D}) \cap C^{\alpha, \frac{\alpha}{2}}(\overline{\mathcal{D}}) .
$$

In case $\mathcal{D}=\Omega \times(0, T)$ with $\Omega=\mathbb{R} \times \Sigma$ or $\Omega=(0,+\infty) \times \Sigma$, we will assume that $a_{i}$ are independent of $x_{1}$, that is, $a_{i}=a_{i}\left(x^{\prime}, t\right), a_{i} \in C^{\alpha, \frac{\alpha}{2}}(\bar{\Sigma} \times[0, T])$. 
Finally, we will say that $u$ is supercaloric if $\mathcal{L} u \leq 0$, and $u$ is subcaloric if $\mathcal{L} u \geq 0$.

\section{Preliminaries on Classical and viscosity solutions}

In this section we give precise definitions of the classical and viscosity solutions and derive some consequences. In particular, we prove that in the situations considered in this paper a classical solution is a viscosity solution.

Definition 2.1. Let $Q=\Omega \times\left(T_{1}, T_{2}\right)$, with $\Omega \subset \mathbb{R}^{N}$ a domain, be a space-time cylinder. Let $v$ be a continuous function in $\bar{Q}$. Then $v$ is called a classical subsolution (supersolution) to $P$ in $Q$ if $v \geq 0$ in $\bar{Q}$ and

(i) $\mathcal{L} v \geq 0 \quad(\leq 0) \quad$ in $Q \cap\{v>0\}$;

(ii) $v \in C^{1}(\overline{\{v>0\}}), \nabla v \in C^{\alpha, \frac{\alpha}{2}}(\overline{\{v>0\}})$;

(iii) for any $(x, t) \in\{v=0\} \cap \partial\{v>0\}$, we have $\nabla v^{+}(x, t) \neq 0$ and

$$
-\frac{\partial v^{+}}{\partial \nu} \geq \sqrt{2 M} \quad(\leq \sqrt{2 M})
$$

where $\nu:=-\frac{\nabla v^{+}}{\left|\nabla v^{+}\right|}$. That is,

$$
\left|\nabla v^{+}\right| \geq \sqrt{2 M} \quad(\leq \sqrt{2 M})
$$

Observe that the set $\{v=0\} \cap \partial\{v>0\}$ is a closed subset of $\bar{Q}$.

We say that $v$ is a classical solution to $P$ in $Q$ if it is both a classical subsolution and a classical supersolution to $P$.

Definition 2.2. Let $u \in C(\bar{Q}) ; u$ is called a viscosity subsolution (supersolution) to $P$ in $Q$ if $u \geq 0$ in $\bar{Q}$ and, for every space-time subcylinder $Q^{\prime} \subset Q$ and for every $v$ bounded classical supersolution (subsolution) to $P$ in $Q^{\prime}$, with $Q^{\prime} \cap \partial\{v>0\}$ bounded,

$$
\begin{aligned}
& u \leq v \quad(u \geq v) \quad \text { on } \partial_{p} Q, \\
& v>0 \quad \text { on } \overline{\{u>0\}} \cap \partial_{p} Q^{\prime}, \\
& \left(u>0 \quad \text { on } \overline{\{v>0\}} \cap \partial_{p} Q^{\prime}\right)
\end{aligned}
$$

implies that $u \leq v(u \geq v)$ in $Q^{\prime}$.

The function $u$ is called a viscosity solution to $P$ if it is both a viscosity supersolution and a viscosity subsolution to $P$.

We can now prove the consistency between both concepts of solution.

Proposition 2.1. If $u$ is a bounded classical supersolution (subsolution) to $P$ in $Q$ with $Q \cap \partial\{u>0\}$ bounded, then $u$ is a viscosity supersolution (subsolution) to $P$ in $Q$.

Proof. Let $Q^{\prime} \subset Q$ be the cylinder $Q^{\prime}=\Omega^{\prime} \times\left(t_{1}, t_{2}\right)$ and let $v$ be a bounded classical subsolution to $P$ in $Q^{\prime}$ with $Q^{\prime} \cap \partial\{v>0\}$ bounded, satisfying

$$
u \geq v \text { on } \partial_{p} Q^{\prime}
$$

and

$$
u>0 \text { on } \overline{\{v>0\}} \cap \partial_{p} Q^{\prime} .
$$

We will show that $u \geq v$ in $Q^{\prime}$.

If $\overline{\{v>0\}} \cap \partial_{p} Q^{\prime}=\emptyset$, then $v=0$ on $\partial_{p} Q^{\prime}$ and therefore $v=0$ in $Q^{\prime}$. Thus, $u \geq v$ in $Q^{\prime}$. 
If $\overline{\{v>0\}} \cap \partial_{p} Q^{\prime} \neq \emptyset$, it follows that $u>0$ in $\overline{\{v>0\}} \cap Q^{\prime}$ for $t_{1} \leq t<\tau$, for some $\tau>t_{1}$. This is a consequence of the continuity of $u$ and $v$, and the fact that the free boundaries of $u$ and $v$ are bounded. It is not hard to see that having $u>0$ in $\overline{\{v>0\}} \cap Q^{\prime}$ for $t_{1} \leq t<s$ implies $u \geq v$ in $Q^{\prime} \cap\left\{t_{1} \leq t \leq s\right\}$. We set

$$
t_{0}=\sup \left\{s: t_{1}<s<t_{2} \text { such that } u>0 \text { in } \overline{\{v>0\}} \cap Q^{\prime} \cap\left\{t_{1} \leq t<s\right\}\right\},
$$

and we will get to a contradiction by assuming $t_{0}<t_{2}$.

We have $t_{0}>t_{1}$ and $u \geq v$ in $Q^{\prime} \cap\left\{t_{1} \leq t \leq t_{0}\right\}$. In addition, there exists a sequence $\left(x_{n}, t_{n}\right) \rightarrow\left(x_{0}, t_{0}\right) \in \overline{Q^{\prime}}$ such that $u\left(x_{n}, t_{n}\right)=0,\left(x_{n}, t_{n}\right) \in \overline{\{v>0\}} \cap Q^{\prime}$. Then, $u\left(x_{0}, t_{0}\right)=v\left(x_{0}, t_{0}\right)=0$ and $\left(x_{0}, t_{0}\right) \in Q^{\prime} \cap \partial\{v>0\}$. Since $v$ is a classical subsolution to $P$, there exists a sequence $y_{n} \rightarrow x_{0}$ such that $0<v\left(y_{n}, t_{0}\right) \leq$ $u\left(y_{n}, t_{0}\right)$, so that we have proved

$$
\begin{aligned}
& u \geq v \text { in } Q^{\prime} \cap\left\{t_{1} \leq t \leq t_{0}\right\}, \\
& \left(x_{0}, t_{0}\right) \in Q^{\prime} \cap \partial\{u>0\} \cap \partial\{v>0\} .
\end{aligned}
$$

That is, the function $u-v$ is positive and supercaloric in $\{v>0\} \cap Q^{\prime} \cap\left\{t_{1}<t \leq t_{0}\right\}$. From the definition of classical subsolution and supersolution we deduce that

$$
\frac{\nabla v^{+}\left(x_{0}, t_{0}\right)}{\left|\nabla v^{+}\left(x_{0}, t_{0}\right)\right|}=\frac{\nabla u^{+}\left(x_{0}, t_{0}\right)}{\left|\nabla u^{+}\left(x_{0}, t_{0}\right)\right|}=-\nu
$$

and from Hopf's principle (see $[\mathrm{KH}]$ )

$$
-u_{\nu}^{+}\left(x_{0}, t_{0}\right)>-v_{\nu}^{+}\left(x_{0}, t_{0}\right) .
$$

But there holds that

$$
\sqrt{2 M} \geq-u_{\nu}^{+}\left(x_{0}, t_{0}\right) \quad \text { and } \quad-v_{\nu}^{+}\left(x_{0}, t_{0}\right) \geq \sqrt{2 M},
$$

which gives a contradiction and proves the result.

Definition 2.3. Let $\Omega \subset \mathbb{R}^{N}$ be a domain and let $Q=\Omega \times(0, T)$. Let $\Gamma_{N}$ be an open $C^{1}$ subset of $\partial \Omega$ and let $\partial_{N} Q=\Gamma_{N} \times(0, T)$.

Let $u \in C(\bar{Q})$. We say that $u$ is a viscosity solution to $P$ in $Q$ with $\frac{\partial u}{\partial \eta}=0$ on $\partial_{N} Q$, if $u \geq 0$ and there holds:

For every space-time subcylinder $Q^{\prime} \subset Q$ and for every $v$ bounded classical supersolution (subsolution) to $P$ in $Q^{\prime}$, with $Q^{\prime} \cap \partial\{v>0\}$ bounded, such that $\frac{\partial v}{\partial \eta}=0$ on $\partial_{p} Q^{\prime} \cap \partial_{N} Q$

$$
\begin{aligned}
u \leq v & (u \geq v) \quad \text { on } \partial_{p} Q^{\prime} \backslash \partial_{N} Q, \\
v>0 & \text { on } \overline{\{u>0\}} \cap \overline{\partial_{p} Q^{\prime} \backslash \partial_{N} Q}, \\
(u>0 & \text { on } \left.\overline{\{v>0\}} \cap \overline{\partial_{p} Q^{\prime} \backslash \partial_{N} Q}\right)
\end{aligned}
$$

implies that $u \leq v(u \geq v)$ in $Q^{\prime}$.

Proposition 2.2. Let $\Omega=\mathbb{R} \times \Sigma($ or $(0,+\infty) \times \Sigma), Q=\Omega \times(0, T)$ and $\partial_{N} Q=$ $\mathbb{R} \times \partial \Sigma \times(0, T) \quad\left(\right.$ or $\left.\partial_{N} Q=(0,+\infty) \times \partial \Sigma \times(0, T)\right)$.

Let $u$ be a bounded classical solution to $P$ in $Q$ with $Q \cap \partial\{u>0\}$ bounded and $\frac{\partial u}{\partial \eta}=0$ on $\partial_{N} Q$. Then $u$ is a viscosity solution to $P$ in $Q$ with $\frac{\partial u}{\partial \eta}=0$ on $\partial_{N} Q$. 
Proof. Let $Q^{\prime} \subset Q$ be the subcylinder $Q^{\prime}=\Omega^{\prime} \times\left(t_{1}, t_{2}\right)$ and let $v$ be a bounded classical subsolution to $P$ in $Q^{\prime}$, with $Q^{\prime} \cap \partial\{v>0\}$ bounded, such that $\frac{\partial v}{\partial \eta}=0$ on $\partial_{p} Q^{\prime} \cap \partial_{N} Q$, satisfying

$$
u \geq v \text { on } \partial_{p} Q^{\prime} \backslash \partial_{N} Q
$$

and

$$
u>0 \text { on } \overline{\{v>0\}} \cap \overline{\partial_{p} Q^{\prime} \backslash \partial_{N} Q} .
$$

We will show that $u \geq v$ in $Q^{\prime}$.

We will proceed as in Proposition 2.1. In fact, we define $t_{0}$ in the same way and there holds that $t_{0}>t_{1}$.

If $t_{0}<t_{2}$, proceeding in a similar way as in Proposition 2.1, we find a point $\left(x_{0}, t_{0}\right)$ such that $u\left(x_{0}, t_{0}\right)=0$ and

$$
\begin{aligned}
& u \geq v \text { in } Q^{\prime} \cap\left\{t_{1} \leq t \leq t_{0}\right\}, \\
& \left(x_{0}, t_{0}\right) \in \partial\{v>0\} \cap\left(Q^{\prime} \cup\left(\partial_{p} Q^{\prime} \cap \partial_{N} Q\right)\right) .
\end{aligned}
$$

If $\left(x_{0}, t_{0}\right) \in Q^{\prime}$ we proceed as in Proposition 2.1 to see that $\left(x_{0}, t_{0}\right) \in \partial\{u>0\}$ and we conclude the proof exactly as the one of Proposition 2.1.

If $\left(x_{0}, t_{0}\right) \in \partial_{N} Q$, necessarily there exists a neighborhood $\mathcal{N}$ of $\left(x_{0}, t_{0}\right)$ such that $\mathcal{N} \cap \partial_{p} Q^{\prime} \subset \partial_{N} Q$ and thus, as in Proposition 2.1 we deduce that $\left(x_{0}, t_{0}\right) \in \partial\{u>0\}$.

Then, it follows that the function $u-v$ is positive and supercaloric in $\mathcal{R}=\{v>$ $0\} \cap Q^{\prime} \cap \mathcal{N} \cap\left\{t_{1}<t \leq t_{0}\right\}$ and $\frac{\partial(u-v)}{\partial \eta}=0$ on $\overline{\{v>0\}} \cap \partial_{N} Q \cap \mathcal{N} \cap\left\{t_{1}<t \leq t_{0}\right\}$. Applying Proposition A.1 and Remark A.1 in the Appendix, we see that we can proceed as in the case in which $\left(x_{0}, t_{0}\right) \in Q^{\prime}$, but considering instead of the operator $\mathcal{L}$, a more general uniformly parabolic operator (see Proposition A.1). And again, the contradiction follows from the application of the result in $[\mathrm{KH}]$.

Observation. The efficiency of the concept of viscosity solution depends on the existence of a sufficient number of classical sub-, super- and solutions to serve as test functions. We recall that, in the case of the heat operator (this is, $a_{i} \equiv 0$ ), the existence of classical solutions has been studied in [Ve, [M], $\mathrm{AG}]$ and [GHV].

As an application of the definition we prove that viscosity subsolutions have the property of finite propagation of the support.

Proposition 2.3. Let $\Omega \subset \mathbb{R}^{N}$ be a bounded domain, $Q=\Omega \times(0, T)$ and $a_{i} \equiv 0$. Given any viscosity subsolution $u$ to $P$ in $Q$ which vanishes at time $t=0$ in an open ball $B_{r}\left(x_{0}\right) \subset \Omega$ and given $r^{\prime} \in(0, r)$ there exists a small time $\tau>0$ such that $u$ vanishes in $B_{r^{\prime}}\left(x_{0}\right)$ for $0<t<\tau$.

Proof. The result is true for classical solutions according to Definition 2.1. For a viscosity subsolution $u$ we only have to compare $u$ with a suitable classical solution, for a small time interval $0 \leq t \leq \tau$. For instance, with a radially-symmetric classical solution $v$, corresponding to initial data $v(x, 0)=f\left(\left|x-x_{0}\right|\right) \geq 0$, such that $f(s)=0$ for $0 \leq s \leq r-\varepsilon, f(s)=2\|u\|_{L^{\infty}}$ for $s \geq r$. By [GHV] this solution exists and thus the conclusion follows.

In the next propositions we will show that, in the situations considered in this paper, a classical solution has a bounded free boundary; and in particular, it is a viscosity solution. 
Proposition 2.4. Let $\Omega=\mathbb{R} \times \Sigma, Q=\Omega \times(0, T), \partial_{N} Q=\mathbb{R} \times \partial \Sigma \times(0, T)$ and $\partial_{D} Q=\partial_{p} Q \backslash \partial_{N} Q$.

Let $u$ be a bounded classical solution to $P$ in $Q$ with $\frac{\partial u}{\partial \eta}=0$ on $\partial_{N} Q$ and $\|u\|_{C^{\alpha, \frac{\alpha}{2}}(\bar{Q})}<\infty$, such that $\left.u\right|_{\partial_{D} Q}$ has a bounded, nonempty free boundary and $u_{x_{1}}<0$ on $\overline{\{u>0\}} \cap \partial_{D} Q$.

Then $Q \cap \partial\{u>0\}$ is bounded.

Proof. From the assumptions it follows that there exist $c, \bar{K}>0$ such that $\left.u\right|_{\partial_{D} Q} \geq$ $c$ for $x_{1}<-\bar{K}$ and $\left.u\right|_{\partial_{D} Q}=0$ for $x_{1}>\bar{K}$.

Let us first consider the case of the heat operator, i.e. $a_{i} \equiv 0$, and let $K=2 \bar{K}$.

On one hand we can see that there exists $\tau_{1}>0$ such that $u(x, t)=0$ for $x_{1}=K$ and $0 \leq t \leq \tau_{1}$. In fact, if not, there exist $\left(x^{n}, t^{n}\right)$ with $u\left(x^{n}, t^{n}\right)>0, x_{1}^{n}=K$ and $t^{n} \rightarrow 0$. Then, there exists $x^{0}$ with $x_{1}^{0}=K$ such that $\left(x^{0}, 0\right) \in \overline{\{u>0\}}$. On the other hand, $u\left(x^{0}, 0\right)=0$. Thus, $\left(x^{0}, 0\right) \in\{u=0\} \cap \partial\{u>0\}$ so that $\left|\nabla u^{+}\left(x^{0}, 0\right)\right|=\sqrt{2 M}>0$. But $u(x, 0)=0$ in a neighborhood of $x^{0}$, which is a contradiction.

On the other hand, there exists $\tau_{2}>0$ such that $u(x, t) \geq \frac{c}{2}$ for $x_{1}<-K$ and $0 \leq t \leq \tau_{2}$. In fact, this is true because $u(x, 0) \geq c$ for $x_{1}<-K$ and $\|u\|_{C^{\alpha, \frac{\alpha}{2}}(\bar{Q})}<\infty$.

It follows that $Q \cap \partial\{u>0\}$ is bounded for $0 \leq t \leq \tau=\min \left\{\tau_{1}, \tau_{2}\right\}$ and therefore, by Proposition 2.2, $u$ is a viscosity solution to $P$ in $Q \cap\{t<\tau\}$ with $\frac{\partial u}{\partial \eta}=0$ on $\partial_{N} Q \cap\{t<\tau\}$.

Let $L=\|u\|_{L^{\infty}(Q)}$ and let

$$
\begin{aligned}
& v_{-}(x, t)=c\left(1-\exp \left\{\frac{\sqrt{2 M}}{c} x_{1}+\frac{2 M}{c^{2}} t+\frac{K \sqrt{2 M}}{c}\right\}\right)^{+}, \\
& v_{+}(x, t)=2 L\left(1-\exp \left\{\frac{\sqrt{2 M}}{2 L} x_{1}+\frac{2 M}{4 L^{2}} t-\frac{K \sqrt{2 M}}{2 L}-\log 2\right\}\right)^{+} .
\end{aligned}
$$

There holds that $v_{ \pm}$are bounded classical solutions to $P$ in $Q$ with $\frac{\partial v_{+}}{\partial \eta}=\frac{\partial v_{-}}{\partial \eta}=0$ on $\partial_{N} Q$ and bounded free boundaries, and

$$
v_{-}(x, 0) \leq u(x, 0) \leq v_{+}(x, 0) .
$$

Moreover,

$$
\begin{aligned}
& u>0 \text { on } \overline{\left\{v_{-}>0\right\}} \cap\{t=0\}, \\
& v_{+}>0 \text { on } \overline{\{u>0\}} \cap\{t=0\} .
\end{aligned}
$$

Therefore,

$$
v_{-}(x, t) \leq u(x, t) \leq v_{+}(x, t) \quad \text { in } Q \cap\{t \leq \tau\}
$$

Let

$$
t_{0}=\sup \left\{\tau>0 / v_{-} \leq u \leq v_{+} \text {in } Q \cap\{t \leq \tau\}\right\} .
$$

Let us see that $t_{0}=T$ (in which case we deduce that $Q \cap \partial\{u>0\}$ is bounded and the proof is finished).

If not, we see that by continuity, $v_{-} \leq u \leq v_{+}$in $Q \cap\left\{t \leq t_{0}\right\}$ and in particular $\Omega \cap \partial\left\{x / u\left(x, t_{0}\right)>0\right\}$ is bounded. Moreover, by proceeding as in the proof of 
Proposition 2.2 it can be seen that

$$
\begin{aligned}
& u>0 \text { on } \overline{\left\{v_{-}>0\right\}} \cap\left\{t=t_{0}\right\}, \\
& v_{+}>0 \text { on } \overline{\{u>0\}} \cap\left\{t=t_{0}\right\} .
\end{aligned}
$$

In addition, since $v_{-}\left(x, t_{0}\right) \leq u\left(x, t_{0}\right) \leq v_{+}\left(x, t_{0}\right)$ there holds that there exist $c_{1}, K_{1}>0$ such that $u \geq c_{1}$ for $x_{1}<-K_{1}, t=t_{0}$ and $u=0$ for $x_{1}>K_{1}$, $t=t_{0}$. Arguing as before, we can see that there exists $t_{1}>t_{0}$ such that $Q \cap\left\{t_{0} \leq\right.$ $\left.t \leq t_{1}\right\} \cap \partial\{u>0\}$ is bounded and therefore, $u$ is a viscosity solution to $P$ in $Q \cap\left\{t_{0}<t<t_{1}\right\}$ with $\frac{\partial u}{\partial \eta}=0$ on $\partial_{N} Q \cap\left\{t_{0}<t<t_{1}\right\}$.

We conclude that

$$
v_{-}(x, t) \leq u(x, t) \leq v_{+}(x, t) \quad \text { in } Q \cap\left\{t \leq t_{1}\right\}
$$

which contradicts the definition of $t_{0}$. Therefore $t_{0}=T$ and the proof is finished for the case $a_{i} \equiv 0$.

When $a_{i} \not \equiv 0$ we replace $v_{-}, v_{+}$by $w_{-}, w_{+}$defined by $w_{-}(x, t)=$ $v_{-}\left(x_{1}+A t, x^{\prime}, t\right)$ and $w_{+}(x, t)=v_{+}\left(x_{1}-A t, x^{\prime}, t\right)$, with $A \geq\left\|a_{1}\right\|_{L^{\infty}}$.

The next propositions can be proved in a similar way as Proposition 2.4 (in the proof of Proposition 2.6 we use Proposition 2.1 instead of Proposition 2.2).

Proposition 2.5. Let $\Omega=(0,+\infty) \times \Sigma, Q=\Omega \times(0, T), \partial_{N} Q=(0,+\infty) \times \partial \Sigma \times$ $(0, T)$ and $\partial_{D} Q=\partial_{p} Q \backslash \partial_{N} Q$.

Let $u$ be a bounded classical solution to $P$ in $Q$ with $\frac{\partial u}{\partial \eta}=0$ on $\partial_{N} Q$, such that $\left.u\right|_{\partial_{D} Q}$ has a bounded, nonempty free boundary and $u_{x_{1}}<0$ on $\overline{\{u>0\}} \cap \partial_{D} Q$.

Assume that $u\left(0, x^{\prime}, t\right)>0$ for $\left(x^{\prime}, t\right) \in \bar{\Sigma} \times[0, T]$. Then $Q \cap \partial\{u>0\}$ is bounded.

Proposition 2.6. Let $\Omega=(0,+\infty) \times \Sigma, Q=\Omega \times(0, T)$ and $\partial_{D} Q=\partial_{p} Q$.

Let $u$ be a bounded classical solution to $P$ in $Q$, such that $\left.u\right|_{\partial_{D} Q}$ has a bounded, nonempty free boundary and $u_{x_{1}}<0$ on $\overline{\{u>0\}} \cap \partial_{D} Q$.

Assume that $u\left(0, x^{\prime}, t\right)>0$ for $\left(x^{\prime}, t\right) \in \bar{\Sigma} \times[0, T]$. Then $Q \cap \partial\{u>0\}$ is bounded.

The same result holds if we let instead $\Omega=\mathbb{R} \times \Sigma$ and we assume that $\|u\|_{C^{\alpha, \frac{\alpha}{2}}(\bar{Q})}$ $<\infty$ (with no assumptions on $u$ on $\{0\} \times \bar{\Sigma} \times[0, T]$ ).

\section{UNIQUENESS OF CLASSICAL AND VISCOSITY SOLUTIONS}

In this section we show that, under suitable assumptions, a classical solution is the unique viscosity solution to the initial and boundary value problem associated to $P$ and, in particular, it is the unique classical solution. This is done in Theorems 3.1 and 3.2 and Corollary 3.1. We also show comparison.

Theorem 3.1. Let $\Omega=(0,+\infty) \times \Sigma, Q=\Omega \times(0, T), \partial_{N} Q=(0,+\infty) \times \partial \Sigma \times(0, T)$ and $\partial_{D} Q=\partial_{p} Q \backslash \partial_{N} Q$.

Let $u$ be a bounded classical solution to $P$ in $Q$ with $\frac{\partial u}{\partial \eta}=0$ on $\partial_{N} Q$, such that $\left.u\right|_{\partial_{D} Q}$ has a bounded, nonempty free boundary and $u_{x_{1}}<0$ on $\overline{\{u>0\}} \cap \partial_{D} Q$.

Assume that $u\left(0, x^{\prime}, t\right)>0$ for $\left(x^{\prime}, t\right) \in \bar{\Sigma} \times[0, T]$ with $u\left(0, x^{\prime}, t\right) \in C^{2,1}(\bar{\Sigma} \times[0, T])$.

Let $v \in C(\bar{Q})$ be a viscosity solution to $P$ in $Q$ with $\frac{\partial v}{\partial \eta}=0$ on $\partial_{N} Q$.

If $v=u$ on $\partial_{D} Q$ and $\overline{\{v>0\}} \cap \partial_{D} Q=\overline{\{u>0\}} \cap \partial_{D} Q$, then $v=u$ in $\bar{Q}$. 
Remark 3.1. Let $u$ and $v$ be as in the statement of Theorem 3.1. The condition $\overline{\{v>0\}} \cap \partial_{D} Q=\overline{\{u>0\}} \cap \partial_{D} Q$ implies that the free boundaries of $u$ and $v$ coincide on $\partial_{D} Q$ and both start from $\Omega \cap \partial\{u(x, 0)>0\}$.

A condition like this one is necessary in order to get the uniqueness result since otherwise, the bounded solution of the heat equation in $Q$ with homogeneous Neumann datum on $\partial_{N} Q$ and with Dirichlet datum $u$ on $\partial_{D} Q$ (which is a viscosity solution to this problem) would be a counterexample when $a_{i} \equiv 0$.

Proof of Theorem 3.1. Let us consider any $v$ as in the statement of the theorem. We will show that $v=u$ in $\bar{Q}$.

Let us first remark that $u$ satisfies the assumptions of Proposition 2.5. Therefore, $Q \cap \partial\{u>0\}$ is bounded.

Let us fix $\delta>0$ small and define, for $(x, t) \in \bar{Q}$,

$$
u_{\delta}(x, t)=u\left(x_{1}+\delta, x^{\prime}, t\right) .
$$

Since $u$ is a classical solution to $P$ in $Q$, then $u$ is a classical subsolution to $P$ in $Q$ and the same happens with $u_{\delta}$. Also $\frac{\partial u_{\delta}}{\partial \eta}=0$ on $\partial_{N} Q$. From our assumptions, it follows that, for $(x, t)$ on $\partial_{D} Q$,

$$
v(x, t)=u(x, t) \geq u\left(x_{1}+\delta, x^{\prime}, t\right)=u_{\delta}(x, t) .
$$

That is,

$$
v \geq u_{\delta} \quad \text { on } \partial_{D} Q
$$

In addition, there holds that

$$
v>0 \quad \text { on } \overline{\left\{u_{\delta}>0\right\}} \cap \partial_{D} Q .
$$

Since $v$ is a viscosity supersolution to $P$ in $Q$ with $\frac{\partial v}{\partial \eta}=0$ on $\partial_{N} Q$, we obtain

$$
v(x, t) \geq u_{\delta}(x, t)=u\left(x_{1}+\delta, x^{\prime}, t\right) \quad \text { for }(x, t) \in \bar{Q},
$$

and letting $\delta \rightarrow 0$ we conclude that

$$
v \geq u \quad \text { in } \bar{Q} .
$$

In order to show that $u \geq v$ in $\bar{Q}$ we proceed in the following way. We first extend $u$ to a neighborhood of $x_{1}=0$ letting, in $-\mu \leq x_{1} \leq 0,\left(x^{\prime}, t\right) \in \bar{\Sigma} \times[0, T]$,

$$
u\left(x_{1}, x^{\prime}, t\right)=u\left(0, x^{\prime}, t\right)-c x_{1}-k x_{1}^{2} .
$$

Here $c>0$ is a constant such that $u_{x_{1}}\left(0^{+}, x^{\prime}, t\right) \leq-c$ for $\left(x^{\prime}, t\right) \in \bar{\Sigma} \times[0, T], k$ is a positive constant and $\mu>0$ is a small constant such that $u>0$ in $-\mu \leq x_{1} \leq 0$.

Then, $u$ is continuous up to $x_{1}=-\mu$ and moreover, it is strictly decreasing in $-\mu \leq x_{1} \leq 0$ in the direction $e_{1}$ if $\mu$ is chosen small enough. Also,

$$
\frac{\partial u}{\partial \eta}=0 \quad \text { on }\left\{-\mu \leq x_{1} \leq 0\right\} \times \partial \Sigma \times(0, T) .
$$

On the other hand, since $u$ is smooth in $-\mu \leq x_{1} \leq 0$ it follows that, if $k$ is large enough and $\mu$ is small, then

$$
\mathcal{L} u \leq 0 \quad \text { in }\left\{-\mu<x_{1}<0\right\} \times \Sigma \times(0, T) .
$$

If we now notice that $u_{x_{1}}\left(0^{-}, x^{\prime}, t\right) \geq u_{x_{1}}\left(0^{+}, x^{\prime}, t\right)$, we deduce that $u$ is supercaloric in $\left\{x_{1}>-\mu\right\} \cap\{u>0\}$. 
Now consider, for $0<\delta<\mu$ small and $(x, t) \in \bar{Q}$,

$$
u^{\delta}\left(x_{1}, x^{\prime}, t\right)=u\left(x_{1}-\delta, x^{\prime}, t\right) .
$$

It is not hard to see that

$$
u^{\delta} \geq v \text { on } \partial_{D} Q
$$

Let us show that $u^{\delta} \geq v$ in $Q$. To this effect let us choose $a>\delta$ such that $u^{\delta}>0$ and $v>0$ in $[0, a] \times \bar{\Sigma} \times[0, T]$. Then $\mathcal{L} v=0$ in $(0, a) \times \Sigma \times(0, T)$ and $\frac{\partial v}{\partial \eta}=0$ on $(0, a) \times \partial \Sigma \times(0, T)$ in the classical sense. This follows from Definition 2.3, using the fact that $v>0$ in this region.

In addition, there holds that $u^{\delta}(x, 0)>v(x, 0)$ in $[0, a] \times \bar{\Sigma}$ and therefore,

$$
u^{\delta}>v \quad \text { in }[0, a] \times \bar{\Sigma} \times[0, \tau],
$$

for some $\tau>0$.

Now let $0<t_{0}<T$ be such that

$$
u^{\delta} \geq v \quad \text { in }[0, a] \times \bar{\Sigma} \times\left[0, t_{0}\right]
$$

We will show that if $a>b>\delta$, then

$$
u^{\delta}>v \quad \text { in }[0, b] \times \bar{\Sigma} \times\left[0, t_{0}\right] .
$$

In fact, $u^{\delta}-v$ is supercaloric in $(0, a) \times \Sigma \times\left(0, t_{0}\right)$ and it is continuous up to the boundary. We also know that $u^{\delta}-v>0$ in $\{0\} \times \Sigma \times[0, T]$ by construction. If we had $u^{\delta}-v=0$ somewhere in $(0, b] \times \Sigma \times\left(0, t_{0}\right]$, we would contradict the strong maximum principle. If, on the other hand, we had $u^{\delta}-v=0$ somewhere on $(0, b] \times \partial \Sigma \times\left(0, t_{0}\right]$, we would contradict the Hopf principle. Then, (3.1) is proved.

From the continuity of $u^{\delta}$ and $v$ it follows that

$$
u^{\delta}>v \quad \text { in }[0, b] \times \bar{\Sigma} \times\left[0, t_{1}\right]
$$

for some $t_{1}>t_{0}$.

Then, $v$ is a viscosity solution to $P$ in $Q$ with $\frac{\partial v}{\partial \eta}=0$ on $\partial_{N} Q$ and $u^{\delta}$ is a classical solution to $P$ in $\left\{x_{1}>b\right\} \cap Q$ with $\frac{\partial u^{\delta}}{\partial \eta}=0$ on $\left\{x_{1}>b\right\} \cap \partial_{N} Q$ satisfying

$$
\begin{array}{ll}
u^{\delta} \geq v & \text { on }\{b\} \times \Sigma \times\left(0, t_{1}\right), \\
u^{\delta} \geq v & \text { on }(b,+\infty) \times \Sigma \times\{0\} \\
u^{\delta}>0 & \text { on } \overline{\{v>0\}} \cap\left(\{t=0\} \cup\left\{x_{1}=b\right\}\right) .
\end{array}
$$

Therefore,

$$
u^{\delta} \geq v \quad \text { in }[0,+\infty) \times \bar{\Sigma} \times\left[0, t_{1}\right] .
$$

Let us now prove that $u^{\delta} \geq v$ in $Q$. If not, we let

$$
\bar{t}=\inf \left\{0 \leq t \leq T / \text { there exists }(x, t) \in Q / u^{\delta}(x, t)<v(x, t)\right\} .
$$

The argument above implies, in particular, that $u^{\delta} \geq v$ if $0 \leq t \leq \tau$ and therefore $0<\bar{t}<T$. From the definition of $\bar{t}$ it follows that

$$
u^{\delta}(x, t) \geq v(x, t) \quad \text { for } t \leq \bar{t} .
$$

If we now let $t_{0}=\bar{t}$ and proceed as above, we deduce that

$$
u^{\delta}(x, t) \geq v(x, t) \quad \text { for } t \leq t_{1},
$$


with $t_{1}>t_{0}=\bar{t}$, a contradiction. Consequently, $u^{\delta} \geq v$ and thus, $u \geq v$. The theorem is proved.

For two classical solutions we have the following uniqueness result.

Corollary 3.1. Let $\Omega, Q, \partial_{N} Q, \partial_{D} Q$ and $u$ as in Theorem 3.1. Let $v$ be a bounded classical solution to $P$ in $Q$ with $\frac{\partial v}{\partial \eta}=0$ on $\partial_{N} Q$, such that $v=u$ on $\partial_{D} Q$. Then, $v=u$ in $\bar{Q}$.

Proof. We first notice that $\overline{\{v>0\}} \cap \partial_{D} Q=\overline{\{u>0\}} \cap \partial_{D} Q$. Then, proceeding as in Proposition 2.5 we deduce $Q \cap \partial\{v>0\}$ is bounded. By Proposition 2.2, $v$ is the viscosity solution to $P$ in $Q$ with $\frac{\partial v}{\partial \eta}=0$ on $\partial_{N} Q$ and thus, from Theorem 3.1 we conclude that $u=v$ in $\bar{Q}$.

A Comparison Principle for bounded classical solutions follows from Proposition 2.2 if the free boundaries are bounded in $Q$ and separated on $\partial_{D} Q$. With a monotonicity assumption on $\partial_{D} Q$, we get a different comparison result.

Corollary 3.2. Let $\Omega, Q, \partial_{N} Q, \partial_{D} Q$ and $u$ as in Theorem 3.1. Let $v$ be a bounded classical solution to $P$ in $Q$ with $\frac{\partial v}{\partial \eta}=0$ on $\partial_{N} Q$, and such that $Q \cap \partial\{v>0\}$ is bounded. If $v \geq u$ on $\partial_{D} Q$, there holds that $v \geq u$ in $\bar{Q}$.

Proof. The proof follows the lines of Theorem 3.1 by using Proposition 2.2.

In the next theorem we prove the uniqueness of viscosity solution under different assumptions from those in Theorem 3.1. As in Corollaries 3.1 and 3.2, uniqueness and comparison of classical solutions follow.

Theorem 3.2. The result of Theorem 3.1 holds if we let instead $\partial_{N} Q=\emptyset$ so that $\partial_{D} Q=\partial_{p} Q$. Moreover, the result of Theorem 3.1 also holds if we let $\Omega=\mathbb{R} \times \Sigma$ with $\partial_{N} Q=\mathbb{R} \times \partial \Sigma \times(0, T)$ or $\partial_{N} Q=\emptyset$, as long as $\|u\|_{C^{\alpha, \frac{\alpha}{2}}(\bar{Q})}<\infty$. In this case we make no assumptions on $u$ on $\{0\} \times \bar{\Sigma} \times[0, T]$.

Proof. The proof follows the lines of the proof of Theorem 3.1. In this case we use Propositions 2.4 and 2.6 instead of Proposition 2.5 in order to see that $Q \cap \partial\{u>0\}$ is bounded.

Corollary 3.3. Let $u$ be as in Theorem 3.1. Then $u$ is a decreasing function in $\bar{Q}$ in the direction $e_{1}$ and $u_{x_{1}}<0$ in $\{u>0\} \cap Q$.

Moreover, for every $\varepsilon>0$, the level set $\{u=\varepsilon\}$ is given by $x_{1}=g_{\varepsilon}\left(x^{\prime}, t\right)$ with $g_{\varepsilon} \in C^{1}(\bar{\Sigma} \times[0, T])$ and $\nabla_{x^{\prime}} g_{\varepsilon} \in C^{\alpha, \frac{\alpha}{2}}(\bar{\Sigma} \times[0, T])$.

The same conclusion holds under the assumptions of Theorem 3.2.

Proof. Let us fix $\delta>0$ small and define, as in Theorem 3.1,

$$
u_{\delta}(x, t)=u\left(x_{1}+\delta, x^{\prime}, t\right) .
$$

Since $u$ is a viscosity solution to $P$ in $Q$ with $\frac{\partial u}{\partial \eta}=0$ on $\partial_{N} Q$, then, reasoning as in Theorem 3.1, we obtain

$$
u(x, t) \geq u_{\delta}(x, t)=u\left(x_{1}+\delta, x^{\prime}, t\right) \quad \text { for }(x, t) \in \bar{Q},
$$

which implies that $u_{x_{1}} \leq 0$ in $\{u>0\}$.

Since $\mathcal{L} u_{x_{1}}=0$ in $Q \cap\{u>0\}$ and $\frac{\partial u_{x_{1}}}{\partial \eta}=0$ on $\{u>0\} \cap \partial_{N} Q$, there holds that, for every $\varepsilon>0, u_{x_{1}}<0$ in $\{u \geq \varepsilon\} \cap\left(Q \cup \partial_{N} Q\right)$. 
Since $u_{x_{1}}<0$ in $\overline{\{u>0\}} \cap \partial_{D} Q$, there holds that for every $\varepsilon>0, u_{x_{1}}<0$ in $\{u \geq \varepsilon\} \cap \bar{Q}$ and the result follows immediately.

\section{Auxiliary Results}

This section contains results on the following problem:

$$
\Delta u-u_{t}=\beta(u)
$$

where the function $\beta$ is as in Section 1. The results will be used in the next sections where $P_{1}$ appears as a blow-up limit. The transport term will disappear in the blow-up process.

Lemma 4.1. Let $a, b \geq 0$ and let $\psi=\psi_{a, b}$ be the classical solution to

$$
\begin{gathered}
\psi_{s s}=\beta(\psi) \quad \text { for } s>0, \\
\psi(0)=a, \quad \psi_{s}(0)=-\sqrt{2 b} .
\end{gathered}
$$

Let $B(\tau)=\int_{0}^{\tau} \beta(\rho) d \rho$.

(4.2) If $b=0$ and $a \in\{0\} \cup[1,+\infty)$, then $\psi \equiv a$.

(4.3) If $b=0$ and $a \in(0,1)$, then $\lim _{s \rightarrow+\infty} \psi(s)=+\infty$.

(4.4) If $b \in(0, B(a))$, then $\lim _{s \rightarrow+\infty} \psi(s)=+\infty$.

(4.5) If $0<b=B(a)$, then $\psi_{s}<0$ and $\lim _{s \rightarrow+\infty} \psi(s)=0$.

(4.6) If $b \in(B(a),+\infty)$, then $\psi_{s}<0$ and $\lim _{s \rightarrow+\infty} \psi(s)=-\infty$.

Proof. We first recall that the function $\beta$ is Lipschitz continuous and therefore, there is a unique classical solution to (4.1).

Let us multiply equation $(4.1)$ by $\psi_{s}$. We get

$$
\psi_{s s} \psi_{s}=\beta(\psi) \psi_{s}=\frac{d}{d s}(B(\psi)) \quad \text { for } s>0
$$

Then, if we integrate the expression above, we deduce that

$$
\frac{1}{2} \psi_{s}^{2}(s)-B(\psi(s))=\frac{1}{2} \psi_{s}^{2}(0)-B(\psi(0))=b-B(a),
$$

for every $s \geq 0$.

I. Assume $b=0$ and $a \in\{0\} \cup[1,+\infty)$. Then, (4.2) follows easily if we recall that $\beta(s)=0$ for $s \in\{0\} \cup[1,+\infty)$.

II. Assume $b=0$ and $a \in(0,1)$. Since $\psi_{s s} \geq 0$, then $\psi_{s}(s) \geq 0$. Moreover, $\psi_{s}(s)>0$ if $s>0$ (otherwise $\psi \equiv a$ in some interval, which is not possible). In particular, given $s_{0}>0$, we must have, for $s>s_{0}$,

$$
\psi(s) \geq \psi\left(s_{0}\right)+\psi_{s}\left(s_{0}\right)\left(s-s_{0}\right)
$$

and hence, (4.3) follows.

III. Assume $b \in(0, B(a))$. From (4.7) we deduce

$$
B(\psi(s)) \geq B(a)-b>0,
$$

which implies, for some constant $\mu$,

$$
\psi(s) \geq \mu>0 .
$$

Let us suppose $a>1$. Then, $\psi_{s s}=\beta(\psi)=0$ near the origin. Hence

$$
\psi(s)=a-\sqrt{2 b} s,
$$


as long as $\psi(s)>1$. In any case $(a>1$ or $a \leq 1)$, there exists $s_{0} \geq 0$ such that $\psi\left(s_{0}\right) \leq 1$ and $\psi_{s}\left(s_{0}\right)=-\sqrt{2 b}$, and therefore, there exists $s_{1} \geq 0$ such that

$$
\psi\left(s_{1}\right)<1, \quad \psi_{s}\left(s_{1}\right)<0 .
$$

If we had $\psi_{s} \leq 0$ for $s \geq s_{1}$, then, from (4.8) and from equation (4.1), we would get, for $s \geq s_{1}$,

$$
0<\mu \leq \psi(s) \leq \psi\left(s_{1}\right)<1 \quad \text { and } \quad \psi_{s s}(s)>\delta>0
$$

for some constant $\delta$. Thus,

$$
0 \geq \psi_{s}(s) \geq \psi_{s}\left(s_{1}\right)+\delta\left(s-s_{1}\right),
$$

for $s \geq s_{1}$, which is not possible.

That is, we have shown that there exists $s_{2}>0$ such that $\psi_{s}\left(s_{2}\right)>0$. Then, $\psi_{s s} \geq 0$ now gives, for $s \geq s_{2}$,

$$
\psi(s) \geq \psi\left(s_{2}\right)+\psi_{s}\left(s_{2}\right)\left(s-s_{2}\right),
$$

that is, (4.4) holds.

IV. Assume $0<b=B(a)$. Now, (4.7) gives

$$
\frac{1}{2} \psi_{s}^{2}(s)=B(\psi(s)) \quad \text { for } s \geq 0 \text {. }
$$

If there existed $s_{0} \geq 0$ such that $\psi_{s}\left(s_{0}\right)=0$, then $B\left(\psi\left(s_{0}\right)\right)=0$, implying $\psi\left(s_{0}\right) \leq 0$. The uniqueness of (4.1) would give $\psi(s) \equiv \psi\left(s_{0}\right)$, a contradiction.

Hence, $\psi_{s}(s)<0$ and thus $B(\psi(s))>0$. This implies that $\psi(s)>0$ and that there exists

$$
\lim _{s \rightarrow+\infty} \psi(s)=\gamma, \quad 0 \leq \gamma<+\infty .
$$

If $\gamma>0$, it follows from (4.9) that $\lim _{s \rightarrow+\infty} \psi_{s}(s)=-\sqrt{2 B(\gamma)}<0$, and then $\psi(s)<0$ for $s$ large. This gives a contradiction and thus, (4.5) holds.

V. Finally, assume $b \in(B(a),+\infty)$. Then, (4.7) gives

$$
\frac{1}{2} \psi_{s}^{2}(s) \geq b-B(a)>0 .
$$

In particular, $\psi_{s}$ never vanishes and we have, $\psi_{s}(s) \leq-\sqrt{2(b-B(a))}$. It follows that

$$
\psi(s) \leq \psi(0)-\sqrt{2(b-B(a))} s,
$$

for $s>0$, then (4.6) holds and the proof is complete.

Lemma 4.2. Let $B(\tau)=\int_{0}^{\tau} \beta(\rho) d \rho$.

a) Let $\psi^{n} \geq 0$, symmetric with respect to $s=\frac{n}{2}$, be a solution to

$$
\begin{aligned}
& \psi_{s s}=\beta(\psi) \quad \text { in }(0, n), \\
& \psi(0)=\psi(n)=a \in(0,1) .
\end{aligned}
$$

Then, $\psi_{s}^{n}(0)=-\sqrt{2 b_{n}}$ with $b_{n} \nearrow B(a)$ as $n \rightarrow \infty$.

b) Let $\psi^{n} \geq 0$ be a solution to

$$
\begin{aligned}
& \psi_{s s}=\beta(\psi) \quad \text { in }(0, n), \\
& \psi(0)=a \in(0,1], \\
& \psi(n)=0 .
\end{aligned}
$$


Then, $\psi_{s}^{n}(0)=-\sqrt{2 b_{n}}$ with $b_{n} \searrow B(a)$ as $n \rightarrow \infty$.

Proof. Part a). Since $\psi^{n}$ is symmetric, $\psi_{s}^{n}\left(\frac{n}{2}\right)=0$.

On the other hand, since

$$
\frac{1}{2}\left(\psi_{s}^{n}\right)^{2}-B\left(\psi^{n}\right)=b_{n}-B(a)
$$

there holds that

$$
-B\left(\psi^{n}(n / 2)\right)=b_{n}-B(a) .
$$

In particular, there holds that $b_{n} \leq B(a)$.

We claim that $\psi^{n}\left(\frac{n}{2}\right) \rightarrow 0$ as $n \rightarrow \infty$. In fact, if not there would exist $\alpha>0$ such that, for a subsequence that we still call $\psi^{n}$,

$$
\psi^{n}(s) \geq \psi^{n}(n / 2) \geq \alpha \quad \text { for } 0 \leq s \leq n .
$$

On the other hand, there holds that $\psi^{n}(s) \leq a$ for $0 \leq s \leq n$. Thus, $\beta\left(\psi^{n}(s)\right) \geq$ $\beta_{0}>0$ for $0 \leq s \leq n$. Therefore, $\psi_{s s}^{n} \geq \beta_{0}$ for $0 \leq s \leq n$ and thus

$$
\psi^{n}(s) \geq \alpha+\frac{\beta_{0}}{2}(s-n / 2)^{2} \quad \text { for } s \in[n / 2, n] .
$$

In particular,

$$
a=\psi^{n}(n) \geq \alpha+\left(\beta_{0} / 8\right) n^{2} \rightarrow \infty \quad \text { as } n \rightarrow \infty
$$

which is a contradiction. Thus,

$$
b_{n}-B(a)=-B\left(\psi^{n}(n / 2)\right) \rightarrow 0 \quad \text { as } n \rightarrow \infty .
$$

Part b). Since

$$
\frac{1}{2}\left(\psi_{s}^{n}\right)^{2}-B\left(\psi^{n}\right)=b_{n}-B(a),
$$

there holds that

$$
\frac{1}{2}\left(\psi_{s}^{n}(n)\right)^{2}=b_{n}-B(a) \geq 0 .
$$

We claim that $\psi_{s}^{n}(n) \rightarrow 0$ as $n \rightarrow \infty$. In fact, if not, there would exist $\alpha>0$ such that, for a subsequence that we still call $\psi^{n}, \psi_{s}^{n}(n) \leq-\alpha$. Since $\psi_{s s}^{n} \geq 0$, there holds that

$$
\psi_{s}^{n}(n) \geq \psi_{s}^{n}(s)
$$

for $0 \leq s \leq n$. Thus,

$$
\psi_{s}^{n}(s) \leq-\alpha \quad \text { for } 0 \leq s \leq n .
$$

Therefore,

$$
a=\psi^{n}(0)=\psi^{n}(0)-\psi^{n}(n)=-\psi_{s}^{n}(\theta) n \geq \alpha n \rightarrow \infty \quad \text { as } n \rightarrow \infty
$$

which is a contradiction. Therefore, $\psi_{s}^{n}(n) \rightarrow 0$ as $n \rightarrow \infty$ and there holds that

$$
b_{n} \rightarrow B(a)
$$

as $n \rightarrow \infty$. 
Lemma 4.3. Let $\mathcal{R}_{\gamma}=\left\{(x, t) \in \mathbb{R}^{N+1} / x_{1}>0,-\infty<t \leq \gamma\right\}, 0 \leq \theta<1$ and let $U \in C^{2+\alpha, 1+\frac{\alpha}{2}}\left(\overline{\mathcal{R}_{\gamma}}\right)$ be such that

$$
\begin{aligned}
\Delta U-U_{t}=\beta(U) & \text { in } \mathcal{R}_{\gamma}, \\
U=1-\theta & \text { on }\left\{x_{1}=0\right\}, \\
0 \leq U \leq 1-\theta & \text { in } \overline{\mathcal{R}_{\gamma} .}
\end{aligned}
$$

1) If $\theta=0$, then $|\nabla U| \leq \sqrt{2 M}$ on $\left\{x_{1}=0\right\}$.

2) If $0<\theta<1$ and $0<\sigma<M$ is such that $\int_{0}^{1-\theta} \beta(\rho) d \rho=M-\sigma$, then $|\nabla U|=\sqrt{2(M-\sigma)}$ on $\left\{x_{1}=0\right\}$.

Proof. For $\theta \in[0,1)$, let $V_{n}$ be the bounded solution to

$$
\begin{aligned}
\Delta V-V_{t} & =\beta(V) \quad \text { in }\left\{0<x_{1}<n, x^{\prime} \in \mathbb{R}^{N-1}, t>0\right\}, \\
V\left(0, x^{\prime}, t\right) & =1-\theta, \\
V\left(n, x^{\prime}, t\right) & =0 \\
V(x, 0) & =0,
\end{aligned}
$$

and let $W_{n}$ be the bounded solution to

$$
\begin{aligned}
\Delta W-W_{t} & =\beta(W) \quad \text { in }\left\{0<x_{1}<n, x^{\prime} \in \mathbb{R}^{N-1}, t>0\right\}, \\
W\left(0, x^{\prime}, t\right) & =1-\theta, \\
W\left(n, x^{\prime}, t\right) & =1-\theta, \\
W(x, 0) & =1-\theta .
\end{aligned}
$$

Let us point out that $V_{n}$ and $W_{n}$ are actually functions of $\left(x_{1}, t\right)$.

For $k \in \mathbb{N}$, let $V_{n}^{k}(x, t)=V_{n}(x, t+k)$ and $W_{n}^{k}(x, t)=W_{n}(x, t+k)$. Since $V_{n}^{k}$, $U$ and $W_{n}^{k}$ are bounded solutions to equation $P_{1}$ in the domain $\left\{0<x_{1}<n, x^{\prime} \in\right.$ $\left.\mathbb{R}^{N-1},-k<t \leq \gamma\right\}$, and on the parabolic boundary of this domain, we have $V_{n}^{k} \leq U \leq W_{n}^{k}$. It follows that

$$
V_{n}^{k}(x, t) \leq U(x, t) \leq W_{n}^{k}(x, t) \quad \text { in }\left\{0<x_{1}<n, x^{\prime} \in \mathbb{R}^{N-1},-k<t \leq \gamma\right\} .
$$

On the other hand (see $[\mathrm{H}]), V_{n}(x, t) \rightarrow \psi_{-}^{n}\left(x_{1}\right)$ uniformly as $t \rightarrow \infty$, where $\psi_{-}^{n} \geq 0$ is a solution to (4.11) with $a=1-\theta$.

Analogously, $W_{n}(x, t) \rightarrow \psi_{+}^{n}\left(x_{1}\right)$ uniformly as $t \rightarrow \infty$, where $\psi_{+}^{n} \geq 0$, symmetric with respect to $x_{1}=\frac{n}{2}$, is a solution to (4.10) with $a=1-\theta$.

Therefore, letting $k \rightarrow \infty$ we get

$$
\psi_{-}^{n}\left(x_{1}\right) \leq U(x, t) \leq \psi_{+}^{n}\left(x_{1}\right) \text { for } 0 \leq x_{1} \leq n, t \leq \gamma .
$$

In particular,

$$
\left(\psi_{-}^{n}\right)_{s}(0) \leq U_{x_{1}}\left(0, x^{\prime}, t\right) \leq\left(\psi_{+}^{n}\right)_{s}(0) \text { for } t \leq \gamma .
$$

Let $\theta=0$. We deduce from Lemma $4.2, \mathrm{~b}$ ) that

$$
-\left|\nabla U\left(0, x^{\prime}, t\right)\right|=U_{x_{1}}\left(0, x^{\prime}, t\right) \geq \lim _{n \rightarrow \infty}\left(\psi_{-}^{n}\right)_{s}(0)=-\sqrt{2 M} .
$$

Let $\theta>0$. We deduce from Lemma 4.2, a) and b) that

$$
-\sqrt{2(M-\sigma)}=\lim _{n \rightarrow \infty}\left(\psi_{-}^{n}\right)_{s}(0) \leq U_{x_{1}}\left(0, x^{\prime}, t\right) \leq \lim _{n \rightarrow \infty}\left(\psi_{+}^{n}\right)_{s}(0)=-\sqrt{2(M-\sigma)} .
$$


Therefore,

$$
-\left|\nabla U\left(0, x^{\prime}, t\right)\right|=U_{x_{1}}\left(0, x^{\prime}, t\right)=-\sqrt{2(M-\sigma)} .
$$

Lemma 4.4. Let $\varepsilon_{j}, \gamma_{\varepsilon_{j}}$ and $\tau_{\varepsilon_{j}}$ be sequences such that $\varepsilon_{j}>0, \varepsilon_{j} \rightarrow 0, \gamma_{\varepsilon_{j}}>0$, $\gamma_{\varepsilon_{j}} \rightarrow \gamma$, with $0 \leq \gamma \leq+\infty, \tau_{\varepsilon_{j}}>0, \tau_{\varepsilon_{j}} \rightarrow \tau$ with $0 \leq \tau \leq+\infty$, and such that $\tau<+\infty$ implies that $\gamma=+\infty$. Let $\rho>0$ and

$$
\mathcal{A}_{\varepsilon_{j}}=\left\{(x, t) /|x|<\frac{\rho}{\varepsilon_{j}},-\min \left(\tau_{\varepsilon_{j}}, \frac{\rho^{2}}{\varepsilon_{j}^{2}}\right)<t<\min \left(\gamma_{\varepsilon_{j}}, \frac{\rho^{2}}{\varepsilon_{j}^{2}}\right)\right\} .
$$

Let $0 \leq \theta<1$ and let $\bar{u}^{\varepsilon_{j}}$ be weak solutions to

$$
\begin{gathered}
\sum_{i, k} \frac{\partial}{\partial x_{i}}\left(a_{i k}^{\varepsilon_{j}}(x) \frac{\partial \bar{u}^{\varepsilon_{j}}}{\partial x_{k}}\right)+\sum_{i} b_{i}^{\varepsilon_{j}}(x, t) \frac{\partial \bar{u}^{\varepsilon_{j}}}{\partial x_{i}}-\bar{u}^{\varepsilon_{j}} \\
=\beta\left(\bar{u}^{\varepsilon_{j}}\right) \text { in }\left\{x_{1}>\bar{f}_{\varepsilon_{j}}\left(x^{\prime}, t\right)\right\} \cap \mathcal{A}_{\varepsilon_{j}}, \\
\bar{u}^{\varepsilon_{j}}=1-\theta \quad \text { on }\left\{x_{1}=\bar{f}_{\varepsilon_{j}}\left(x^{\prime}, t\right)\right\} \cap \mathcal{A}_{\varepsilon_{j}}, \\
0 \leq \bar{u}^{\varepsilon_{j}} \leq 1-\theta \quad \text { in }\left\{x_{1} \geq \bar{f}_{\varepsilon_{j}}\left(x^{\prime}, t\right)\right\} \cap \overline{\mathcal{A}_{\varepsilon_{j}}},
\end{gathered}
$$

with $\bar{u}^{\varepsilon_{j}} \in C\left(\left\{x_{1} \geq \bar{f}_{\varepsilon_{j}}\left(x^{\prime}, t\right)\right\} \cap \overline{\mathcal{A}_{\varepsilon_{j}}}\right)$, and $\nabla \bar{u}^{\varepsilon_{j}} \in L^{2}$. Here $a_{i k}^{\varepsilon_{j}} \rightarrow \delta_{i k}$ and $b_{i}^{\varepsilon_{j}} \rightarrow 0$ uniformly on compact sets of $\mathbb{R}^{N}$ and of $\mathbb{R}^{N} \times(-\tau, \gamma)$ respectively, and $\bar{f}_{\varepsilon_{j}}$ are continuous functions such that $\bar{f}_{\varepsilon_{j}}(0,0)=0$ with $\bar{f}_{\varepsilon_{j}} \rightarrow 0$ uniformly on compact subsets of $\mathbb{R}^{N-1} \times(-\tau, \gamma)$. Moreover, we assume that $\left\|\bar{f}_{\varepsilon_{j}}\right\|_{C^{1}(K)}+\left\|\nabla_{x^{\prime}} \bar{f}_{\varepsilon_{j}}\right\|_{C^{\alpha, \frac{\alpha}{2}}(K)}$ are uniformly bounded, for every compact set $K \subset \mathbb{R}^{N-1} \times(-\tau, \gamma)$, and in addition, $\left\|b_{i}^{\varepsilon_{j}}\right\|_{L^{\infty}}$ and $\left\|a_{i k}^{\varepsilon_{j}}\right\|_{W^{1, \infty}}$ are uniformly bounded. Moreover, $a_{i k}^{\varepsilon_{j}}$ are uniformly parabolic with constant independent of $\varepsilon_{j}$.

Then, there exists a function $\bar{u}$ such that, for a subsequence,

$$
\begin{aligned}
& \bar{u} \in C^{2+\alpha, 1+\frac{\alpha}{2}}\left(\left\{x_{1} \geq 0, \gamma>t>-\tau\right\}\right), \\
\bar{u}^{\varepsilon_{j}} \rightarrow \bar{u} & \text { uniformly on compact subsets of }\left\{x_{1}>0, \gamma>t>-\tau\right\}, \\
\Delta \bar{u}-\bar{u}_{t}=\beta(\bar{u}) & \text { in }\left\{x_{1}>0, \gamma>t>-\tau\right\}, \\
\bar{u}=1-\theta & \text { on }\left\{x_{1}=0, \gamma>t>-\tau\right\}, \\
0 \leq \bar{u} \leq 1-\theta & \text { in }\left\{x_{1} \geq 0, \gamma>t>-\tau\right\} .
\end{aligned}
$$

If $\gamma<+\infty$, we require, in addition, that

$$
\left\|\bar{f}_{\varepsilon_{j}}\left(x^{\prime}, t+\gamma_{\varepsilon_{j}}-\gamma\right)\right\|_{C^{1}(K)}+\left\|\nabla_{x^{\prime}} \bar{f}_{\varepsilon_{j}}\left(x^{\prime}, t+\gamma_{\varepsilon_{j}}-\gamma\right)\right\|_{C^{\alpha, \frac{\alpha}{2}}(K)}
$$

be uniformly bounded for every compact set $K \subset \mathbb{R}^{N-1} \times(-\infty, \gamma]$. And we deduce that

$$
\bar{u} \in C^{2+\alpha, 1+\frac{\alpha}{2}}\left(\left\{x_{1} \geq 0, t \leq \gamma\right\}\right) .
$$

If $\tau<+\infty$, we let

$$
\mathcal{B}_{\varepsilon_{j}}=\left\{x /|x|<\frac{\rho}{\varepsilon_{j}}, x_{1}>\bar{f}_{\varepsilon_{j}}\left(x^{\prime},-\tau_{\varepsilon_{j}}\right)\right\},
$$

and we require, in addition, that for every $R>0$,

$$
\left\|\bar{u}^{\varepsilon_{j}}\left(x,-\tau_{\varepsilon_{j}}\right)\right\|_{C^{\alpha}\left(\overline{\mathcal{B}}_{\varepsilon_{j}} \cap \bar{B}_{R}(0)\right)} \leq C_{R}
$$


and that there exists $r>0$ such that

$$
\left\|\bar{u}^{\varepsilon_{j}}\left(x,-\tau_{\varepsilon_{j}}\right)\right\|_{C^{1+\alpha}\left(\overline{\mathcal{B}}_{\varepsilon_{j}} \cap \bar{B}_{r}(0)\right)} \leq C_{r} .
$$

Moreover, we assume that $\left\|\bar{f}_{\varepsilon_{j}}\left(x^{\prime}, t-\tau_{\varepsilon_{j}}+\tau\right)\right\|_{C^{1}(K)}+\left\|\nabla_{x^{\prime}} \bar{f}_{\varepsilon_{j}}\left(x^{\prime}, t-\tau_{\varepsilon_{j}}+\tau\right)\right\|_{C^{\alpha, \frac{\alpha}{2}}(K)}$ are uniformly bounded for every compact set $K \subset \mathbb{R}^{N-1} \times[-\tau,+\infty)$.

Then, there holds that

$$
\begin{aligned}
& \bar{u} \in C^{\alpha, \frac{\alpha}{2}}\left(\left\{x_{1} \geq 0, t \geq-\tau\right\}\right), \nabla \bar{u} \in C\left(\left\{0 \leq x_{1}<r, t \geq-\tau\right\}\right), \\
& \bar{u}^{\varepsilon_{j}}\left(x,-\tau_{\varepsilon_{j}}\right) \rightarrow \bar{u}(x,-\tau) \quad \text { uniformly on compact subsets of }\left\{x_{1}>0\right\} .
\end{aligned}
$$

In any case $(\tau, \gamma$ be infinite or finite)

$$
\left|\nabla \bar{u}^{\varepsilon_{j}}(0,0)\right| \rightarrow|\nabla \bar{u}(0,0)| .
$$

Proof. We will drop the subscript $j$ when referring to the sequences defined in the statement and $\varepsilon \rightarrow 0$ will mean $j \rightarrow \infty$.

Case I. $\tau=+\infty, \gamma=+\infty$.

In order to prove the result, we first apply suitable changes of variables to straighten up the boundaries $x_{1}=\bar{f}_{\varepsilon}\left(x^{\prime}, t\right)$. Namely, for every $\varepsilon$, we let

$$
z=h^{\varepsilon}(x, t)
$$

where

$$
h_{1}^{\varepsilon}=x_{1}-\bar{f}_{\varepsilon}\left(x^{\prime}, t\right), \quad h_{i}^{\varepsilon}=x_{i}, \quad i>1,
$$

and we define

$$
\bar{v}^{\varepsilon}(z, t)=\bar{u}^{\varepsilon}(x, t) .
$$

Let $R>0$ be fixed and let

$$
B_{R}^{+}=\left\{(z, t) / z_{1}>0\right\} \cap B_{R}(0,0) .
$$

Then the function $\bar{v}^{\varepsilon} \in C\left(\overline{B_{R}^{+}}\right)$, with $\nabla \bar{v}^{\varepsilon} \in L^{2}\left(B_{R}^{+}\right)$is a weak solution to

$$
\begin{array}{rlrl}
\sum_{r, l} \frac{\partial}{\partial z_{r}}\left(\tilde{a}_{r l}^{\varepsilon}(z, t) \frac{\partial \bar{v}^{\varepsilon}}{\partial z_{l}}\right)+\sum_{r} \tilde{b}_{r}^{\varepsilon}(z, t) \frac{\partial \bar{v}^{\varepsilon}}{\partial z_{r}}-\frac{\partial \bar{v}^{\varepsilon}}{\partial t}=\beta\left(\bar{v}^{\varepsilon}\right) & \text { in } B_{R}^{+}, \\
\bar{v}^{\varepsilon}=1-\theta & & \text { on } \overline{B_{R}^{+}} \cap\left\{z_{1}=0\right\}, \\
0 \leq \bar{v}^{\varepsilon} \leq 1-\theta & & \text { in } \overline{B_{R}^{+}}
\end{array}
$$

if $\varepsilon$ is small enough. Here

$$
\begin{aligned}
& \tilde{a}_{r l}^{\varepsilon}(z, t)=\sum_{i, k} a_{i k}^{\varepsilon}(x) \frac{\partial h_{r}^{\varepsilon}}{\partial x_{i}} \frac{\partial h_{l}^{\varepsilon}}{\partial x_{k}}, \\
& \tilde{b}_{r}^{\varepsilon}(z, t)=\sum_{i} b_{i}^{\varepsilon}(x, t) \frac{\partial h_{r}^{\varepsilon}}{\partial x_{i}}-\frac{\partial h_{r}^{\varepsilon}}{\partial t} .
\end{aligned}
$$

Note that there exists $C_{R}>0$ such that

$$
\begin{aligned}
& \left\|\tilde{a}_{r l}^{\varepsilon}\right\|_{C^{\alpha, \frac{\alpha}{2}}\left(\overline{B_{R}^{+}}\right)} \leq C_{R}, \\
& \left\|\tilde{b}_{r}^{\varepsilon}\right\|_{L^{\infty}\left(B_{R}^{+}\right)} \leq C_{R} .
\end{aligned}
$$


Moreover, there exists $\lambda^{\prime}>0$ such that, if $\varepsilon$ is small enough,

$$
\sum_{r, l} \tilde{a}_{r l}^{\varepsilon}(z, t) \xi_{r} \xi_{l} \geq \lambda^{\prime}|\xi|^{2} \quad \text { for }(z, t) \in \overline{B_{R}^{+}} .
$$

Here we have used that, by hypothesis, there exists $\lambda>0$ such that

$$
\sum_{i, k} a_{i k}^{\varepsilon}(x) \xi_{i} \xi_{k} \geq \lambda|\xi|^{2}
$$

and the fact that $\left|\left(D h^{\varepsilon}\right)^{-1}\right|$ are uniformly bounded on any compact set, if $\varepsilon$ is small enough.

By Theorem 10.1, Chapter III in [LSU], there exists $C_{R}>0$ such that

$$
\left\|\bar{v}^{\varepsilon}\right\|_{C^{\alpha, \frac{\alpha}{2}}\left(\overline{B_{\frac{R}{2}}^{+}}\right)} \leq C_{R}
$$

On the other hand, by Theorem 1.4.3 in [CK] we also have that

$$
\left\|\nabla \bar{v}^{\varepsilon}\right\|_{L^{\infty}\left(\overline{B_{\frac{R}{2}}^{+}}\right)} \leq C_{R}
$$

Moreover, by Theorem 1.4.10 in $\left[\mathrm{CK}\right.$, the functions $\nabla \bar{v}^{\varepsilon}$ are continuous in $\overline{B_{\frac{R}{2}}^{+}}$with a modulus of continuity independent of $\varepsilon$.

Therefore, there exists a function $\bar{u} \in C^{\alpha, \frac{\alpha}{2}}\left(\overline{B_{\frac{R}{2}}^{+}}\right)$and a subsequence that we still call $\bar{v}^{\varepsilon}$ such that $\bar{v}^{\varepsilon} \rightarrow \bar{u}$ and $\nabla \bar{v}^{\varepsilon} \rightarrow \nabla \bar{u}$ uniformly in $\overline{B_{\frac{R}{2}}^{+}}$.

Clearly,

$$
\begin{aligned}
\bar{u}=1-\theta & \text { in }\left\{z_{1}=0\right\} \cap \overline{B_{\frac{R}{2}}^{+}}, \\
0 \leq \bar{u} \leq 1-\theta & \text { in } \overline{B_{\frac{R}{2}}^{+}} .
\end{aligned}
$$

Since $\bar{f}_{\varepsilon} \rightarrow 0$ and $\nabla_{x^{\prime}} \bar{f}_{\varepsilon} \rightarrow 0$ uniformly on compact sets, it is easy to see that we actually have that

$$
\bar{u}^{\varepsilon} \rightarrow \bar{u} \quad \text { uniformly on compact sets of } B_{\frac{R}{2}}^{+}
$$

and

$$
\nabla \bar{u}^{\varepsilon} \rightarrow \nabla \bar{u} \quad \text { uniformly on compact sets of } B_{\frac{R}{2}}^{+} .
$$

Clearly $\bar{u}$ is a solution of $\Delta \bar{u}-\bar{u}_{t}=\beta(\bar{u})$ in $B_{\frac{R}{2}}^{+}$. Standard Schauder estimates imply that $\bar{u} \in C^{2+\alpha, 1+\frac{\alpha}{2}}\left(\overline{B_{\frac{R}{4}}^{+}}\right)$.

Since $\bar{f}_{\varepsilon}(0,0)=0, \nabla_{x^{\prime}} \bar{f}_{\varepsilon}(0,0) \rightarrow 0$ and $\nabla \bar{v}^{\varepsilon}(0,0) \rightarrow \nabla \bar{u}(0,0)$, it is easy to see that $\nabla \bar{u}^{\varepsilon}(0,0) \rightarrow \nabla \bar{u}(0,0)$.

Since $R$ is arbitrary, a standard procedure gives the result in

$$
\left\{z_{1}>0,-\tau<t<\gamma\right\} \quad \text { for } \tau=+\infty \text { and } \gamma=+\infty .
$$

Case II. $\tau<+\infty$.

As in the previous case, we apply suitable changes of variables to straighten up the boundaries $x_{1}=\bar{f}_{\varepsilon}\left(x^{\prime}, t\right)$. Namely, for every $\varepsilon$, we let

$$
\left\{\begin{array}{l}
z=h^{\varepsilon}(x, t), \\
s=t+\tau_{\varepsilon}-\tau
\end{array}\right.
$$


where

$$
h_{1}^{\varepsilon}(x, t)=x_{1}-\bar{f}_{\varepsilon}\left(x^{\prime}, t\right), \quad h_{i}^{\varepsilon}(x, t)=x_{i}, \quad i>1,
$$

and we define

$$
\bar{w}^{\varepsilon}(z, s)=\bar{u}^{\varepsilon}(x, t) .
$$

Let $R>0$ be fixed and let

$$
B_{R, \tau}^{+}=\left\{(z, s) / z_{1}>0, s>-\tau\right\} \cap B_{R}(0,0) .
$$

Then the function $\bar{w}^{\varepsilon} \in C\left(\overline{B_{R, \tau}^{+}}\right)$, with $\nabla \bar{w}^{\varepsilon} \in L^{2}\left(B_{R, \tau}^{+}\right)$is a weak solution to

$$
\begin{array}{rlrl}
\sum_{r, l} \frac{\partial}{\partial z_{r}}\left(\tilde{a}_{r l}^{\varepsilon}(z, s) \frac{\partial \bar{w}^{\varepsilon}}{\partial z_{l}}\right)+\sum_{r} \tilde{b}_{r}^{\varepsilon}(z, s) & \frac{\partial \bar{w}^{\varepsilon}}{\partial z_{r}}-\frac{\partial \bar{w}^{\varepsilon}}{\partial s}=\beta\left(\bar{w}^{\varepsilon}\right) & \text { in } B_{R, \tau}^{+}, \\
\bar{w}^{\varepsilon}=1-\theta & \text { on } \overline{B_{R, \tau}^{+}} \cap\left\{z_{1}=0\right\}, \\
0 \leq \bar{w}^{\varepsilon} \leq 1-\theta & \text { in } \overline{B_{R, \tau}^{+}}, \\
\bar{w}^{\varepsilon}=g^{\varepsilon}(z) & \text { in } \overline{B_{R, \tau}^{+}} \cap\{s=-\tau\} &
\end{array}
$$

if $\varepsilon$ is small enough, where we have called $g^{\varepsilon}(z)=\bar{w}^{\varepsilon}(z,-\tau)$. Here $\tilde{a}_{r l}^{\varepsilon}(z, s)$ and $\tilde{b}_{r}^{\varepsilon}(z, s)$ are defined in $B_{R, \tau}^{+}$in a way analogous to (4.12) and moreover, they satisfy estimates similar to those in (4.13) and (4.14) in $B_{R, \tau}^{+}$. In addition,

$$
\left\|g^{\varepsilon}\right\|_{C^{\alpha}\left(\overline{B_{R}(0)} \cap\left\{z_{1} \geq 0\right\}\right)} \leq C_{R} \quad \text { and } \quad\left\|g^{\varepsilon}\right\|_{C^{1+\alpha}\left(\overline{B_{r}(0)} \cap\left\{z_{1} \geq 0\right\}\right)} \leq C_{r} .
$$

Moreover, $g^{\varepsilon}=1-\theta$ on $\left\{z_{1}=0\right\}$.

By Theorem 10.1, Chapter III in [LSU], there exists $C_{R}>0$ such that

$$
\left\|\bar{w}^{\varepsilon}\right\|_{C^{\alpha, \frac{\alpha}{2}}}\left(\overline{\left.B_{\frac{R}{2}, \tau}^{+}\right)} \leq C_{R} .\right.
$$

On the other hand, by Remark 1.4.11 in [CK], applied to the functions $\hat{w}^{\varepsilon}=\bar{w}^{\varepsilon}-g^{\varepsilon}$, we also have that

$$
\left\|\nabla \bar{w}^{\varepsilon}\right\|_{L^{\infty}\left(\left(\overline{B_{\frac{r}{2}}}(0) \times\left[-\tau, \frac{R}{2}\right]\right) \cap\left\{z_{1} \geq 0\right\}\right)} \leq C_{R}
$$

and that the functions $\nabla \bar{w}^{\varepsilon}$ are continuous in $\left(\overline{B_{\frac{r}{2}}}(0) \times\left[-\tau, \frac{R}{2}\right]\right) \cap\left\{z_{1} \geq 0\right\}$ with a modulus of continuity independent of $\varepsilon$.

Proceeding as in the case $\tau=+\infty$ and using that $\tau_{\varepsilon} \rightarrow \tau$ we see that there exists a function $\bar{u} \in C^{\alpha, \frac{\alpha}{2}}\left(\overline{B_{\frac{R}{2}, \tau}^{+}}\right)$such that for a subsequence

$\bar{w}^{\varepsilon} \rightarrow \bar{u} \quad$ uniformly in $\overline{B_{\frac{R}{2}, \tau}^{+}}$,

$\nabla \bar{w}^{\varepsilon} \rightarrow \nabla \bar{u} \quad$ uniformly on compact sets of $B_{\frac{R}{2}, \tau}^{+}$,

$\bar{u}^{\varepsilon} \rightarrow \bar{u}, \quad \nabla \bar{u}^{\varepsilon} \rightarrow \nabla \bar{u} \quad$ uniformly on compact sets of $B_{\frac{R}{2}, \tau}^{+}$,

$\bar{u}^{\varepsilon}\left(z,-\tau_{\varepsilon}\right) \rightarrow \bar{u}(z,-\tau)$ uniformly on compact sets of $\left\{z_{1}>0\right\} \cap B_{\frac{R}{2}}(0)$,

$\nabla \bar{w}^{\varepsilon} \rightarrow \nabla \bar{u} \quad$ uniformly in $\left(\overline{B_{\frac{r}{2}}}(0) \times\left[-\tau, \frac{R}{2}\right]\right) \cap\left\{z_{1} \geq 0\right\}$. 
This function $\bar{u}$ satisfies

$$
\begin{aligned}
\bar{u} \in C^{2+\alpha, 1+\frac{\alpha}{2}} & \left(\left\{z_{1} \geq 0, t>-\tau\right\} \cap B_{\frac{R}{2}}(0,0)\right), \\
\Delta \bar{u}-\bar{u}_{t}=\beta(\bar{u}) & \text { in }\left\{z_{1}>0, t>-\tau\right\} \cap B_{\frac{R}{2}}(0,0), \\
\bar{u}=1-\theta & \text { on }\left\{z_{1}=0, t \geq-\tau\right\} \cap B_{\frac{R}{2}}(0,0), \\
0 \leq \bar{u} \leq 1-\theta & \text { in }\left\{z_{1} \geq 0, t \geq-\tau\right\} \cap B_{\frac{R}{2}}(0,0) .
\end{aligned}
$$

Moreover, there holds that $\nabla \bar{u}^{\varepsilon}(0,0) \rightarrow \nabla \bar{u}(0,0)$.

Since $R$ is arbitrary, Case II is proved.

Case III. $\gamma<+\infty$.

We proceed as in the previous cases. For every $\varepsilon$, we let

$$
\left\{\begin{array}{l}
z=h^{\varepsilon}(x, t) \\
s=t-\gamma_{\varepsilon}+\gamma
\end{array}\right.
$$

where

$$
h_{1}^{\varepsilon}(x, t)=x_{1}-\bar{f}_{\varepsilon}\left(x^{\prime}, t\right), \quad h_{i}^{\varepsilon}(x, t)=x_{i}, \quad i>1,
$$

and we define

$$
\bar{w}^{\varepsilon}(z, s)=\bar{u}^{\varepsilon}(x, t) .
$$

Let $R>0$ be fixed and let

$$
B_{R, \gamma}^{+}=\left\{(z, s) / z_{1}>0, s<\gamma\right\} \cap B_{R}(0,0) .
$$

As in the previous cases, by using Theorem 10.1, Chapter III in [LSU], and Theorems 1.4.3 and 1.4.10 in $[\mathrm{CK}]$ we deduce that there exists a function $\bar{u} \in C^{\alpha, \frac{\alpha}{2}}\left(\overline{B_{\frac{R}{2}, \gamma}^{+}}\right)$ such that for a subsequence

$$
\begin{aligned}
& \bar{w}^{\varepsilon} \rightarrow \bar{u}, \quad \nabla \bar{w}^{\varepsilon} \rightarrow \nabla \bar{u} \quad \text { uniformly in } \overline{B_{\frac{R}{2}, \gamma}^{+}} \\
& \bar{u}^{\varepsilon} \rightarrow \bar{u}, \quad \nabla \bar{u}^{\varepsilon} \rightarrow \nabla \bar{u} \quad \text { uniformly on compact sets of } B_{\frac{R}{2}, \gamma}^{+} .
\end{aligned}
$$

This function $\bar{u}$ satisfies

$$
\begin{aligned}
& \bar{u} \in C^{2+\alpha, 1+\frac{\alpha}{2}}\left(\left\{z_{1} \geq 0, t \leq \gamma\right\} \cap B_{\frac{R}{2}}(0,0)\right), \\
& \Delta \bar{u}-\bar{u}_{t}=\beta(\bar{u}) \text { in }\left\{z_{1}>0, t<\gamma\right\} \cap B_{\frac{R}{2}}(0,0), \\
& \bar{u}=1-\theta \text { on }\left\{z_{1}=0, t \leq \gamma\right\} \cap B_{\frac{R}{2}}(0,0), \\
& 0 \leq \bar{u} \leq 1-\theta \text { in }\left\{z_{1} \geq 0, t \leq \gamma\right\} \cap B_{\frac{R}{2}}(0,0) .
\end{aligned}
$$

Moreover, there holds that $\nabla \bar{u}^{\varepsilon}(0,0) \rightarrow \nabla \bar{u}(0,0)$.

Since $R$ is arbitrary, the lemma is proved.

\section{Approximation Results}

In this section we prove that, under certain assumptions, a classical subsolution to problem $P$ is the uniform limit of a family of subsolutions to problem $P_{\varepsilon}$ (Theorem 5.1). We prove the analogous result for supersolutions (Theorem 5.2).

Throughout this section we will assume that $\Omega=\mathbb{R} \times \Sigma($ or $\Omega=(0,+\infty) \times \Sigma)$. We define $Q=\Omega \times(0, T)$ and we let $\partial_{N} Q=\mathbb{R} \times \partial \Sigma \times(0, T)$ (or $\partial_{N} Q=(0,+\infty) \times$ $\partial \Sigma \times(0, T))$. In addition, $w$ will be a function satisfying 
(H1)

i) For every $\varepsilon>0$ small, $\{w>\varepsilon\}$ is given by $x_{1}<g_{\varepsilon}\left(x^{\prime}, t\right)$ with $g_{\varepsilon} \in$ $C^{1}(\bar{\Sigma} \times[0, T])$ and $\nabla_{x^{\prime}} g_{\varepsilon} \in C^{\alpha, \frac{\alpha}{2}}(\bar{\Sigma} \times[0, T])$. Moreover, $\left\|g_{\varepsilon}\right\|_{L^{\infty}(\Sigma \times(0, T))}+$ $\left\|g_{\varepsilon}\left(x^{\prime}, 0\right)\right\|_{C^{\alpha}(\bar{\Sigma})} \leq C$ for $\varepsilon$ small.

ii) $w_{x_{1}}<0$ on $\overline{\{w>0\}} \cap\{t=0\}$.

iii) In case $\Omega=(0,+\infty) \times \Sigma$, we assume that $w\left(0, x^{\prime}, t\right)>0$ for $\left(x^{\prime}, t\right) \in \bar{\Sigma} \times[0, T]$.

Theorem 5.1. Let $w$ be a classical subsolution to $P$ in $Q$, with $\frac{\partial w}{\partial \eta}=0$ on $\partial_{N} Q$, satisfying $(\mathbf{H 1})$. Assume, in addition, that there exists $\delta_{0}>0$ such that

$$
\left|\nabla w^{+}\right|=\sqrt{2 M+\delta_{0}} \quad \text { on } Q \cap \partial\{w>0\} .
$$

Let $R>0$. Then, there exists a family $v^{\varepsilon} \in C(\bar{Q})$, with $\nabla v^{\varepsilon} \in L_{\text {loc }}^{2}(\bar{Q})$, of weak subsolutions to $P_{\varepsilon}$ in $Q$, with $\frac{\partial v^{\varepsilon}}{\partial \eta}=0$ on $\partial_{N} Q$, such that, as $\varepsilon \rightarrow 0, v^{\varepsilon} \rightarrow w$ uniformly in $\bar{Q}$.

Moreover, $v^{\varepsilon}=w$ in $\{w \geq \varepsilon\}, v^{\varepsilon}=0$ in $\left\{x_{1} \geq g_{\varepsilon}\left(x^{\prime}, t\right)+R\right\}$ and $\nabla v^{\varepsilon} \in$ $C\left(\bar{Q} \cap\left\{g_{\varepsilon}\left(x^{\prime}, t\right) \leq x_{1} \leq g_{\varepsilon}\left(x^{\prime}, t\right)+R\right\} \cap\{t>0\}\right)$.

Proof. Step I. Construction of the family $v^{\varepsilon}$. For every $\varepsilon>0$ small, we define the domain $D_{R}^{\varepsilon} \subset Q$ in the following way:

$$
D_{R}^{\varepsilon}=\left\{(x, t) \in Q / g_{\varepsilon}\left(x^{\prime}, t\right)<x_{1}<g_{\varepsilon}\left(x^{\prime}, t\right)+R\right\} .
$$

Let $w^{\varepsilon}$ be the solution to $P_{\varepsilon}$ in $D_{R}^{\varepsilon}$ with boundary data:

$$
\begin{aligned}
w^{\varepsilon}(x, t) & = \begin{cases}\varepsilon & \text { on } x_{1}=g_{\varepsilon}\left(x^{\prime}, t\right), \\
0 & \text { on } x_{1}=g_{\varepsilon}\left(x^{\prime}, t\right)+R, \\
w_{0}^{\varepsilon}(x) & \text { on } \partial D_{R}^{\varepsilon} \cap\{t=0\},\end{cases} \\
\frac{\partial w^{\varepsilon}}{\partial \eta}=0 & \text { on } \partial_{N} D_{R}^{\varepsilon}:=\partial_{N} Q \cap\left\{g_{\varepsilon}\left(x^{\prime}, t\right)<x_{1}<g_{\varepsilon}\left(x^{\prime}, t\right)+R\right\} .
\end{aligned}
$$

In order to give the initial data $w_{0}^{\varepsilon}$ we let $\psi=\psi_{a, b}$ be the solution to (4.1) with

$$
a=1, \quad b=M+\delta_{0} / 8 .
$$

We now let

$$
\varphi(\xi)=\psi^{+}\left(\frac{1-\xi}{\sqrt{2 M+\delta_{0}}}\right)
$$

and we define

$$
w_{0}^{\varepsilon}(x)=\varepsilon \varphi\left(\frac{1}{\varepsilon} w(x, 0)\right) \chi(\tilde{w}(x))
$$

where $\tilde{w}$ is a locally Lipschitz continuous function in $\bar{\Omega}$ such that $\tilde{w}(x)=w(x, 0)$ in $\{w(x, 0)>0\}$ and $\tilde{w}<0$ in $\bar{\Omega} \backslash \overline{\{w(x, 0)>0\}}$. Here $\chi \in C^{\infty}(\mathbb{R})$ is such that $\chi(s) \equiv 1$ for $s>-\frac{1}{3} k, \chi(s) \equiv 0$ for $s<-\frac{2}{3} k, 0 \leq \chi \leq 1$ and $-k=\max \left\{\tilde{w}(x)\right.$, for $x / x_{1}=$ $\left.g_{0}\left(x^{\prime}, 0\right)+R\right\}$ where $g_{0}\left(x^{\prime}, 0\right)=\lim _{\varepsilon \rightarrow 0} g_{\varepsilon}\left(x^{\prime}, 0\right)$ uniformly on $\bar{\Sigma}$. Note that $\{w=$ $0\} \cap \partial\{w>0\} \cap\{t=0\}$ is given by $x_{1}=g_{0}\left(x^{\prime}, 0\right)$.

For the existence and regularity of such a solution we refer to [LVW], Theorem 1.1, where it is shown that there exists a unique solution $w^{\varepsilon} \in C\left(\overline{D_{R}^{\varepsilon}}\right)$ with $\nabla w^{\varepsilon} \in$ $C\left(\overline{D_{R}^{\varepsilon}} \cap\{t>0\}\right) \cap L^{2}\left(D_{R}^{\varepsilon}\right)$. 
Finally, we define the family $v^{\varepsilon}$ as follows:

$$
v^{\varepsilon}= \begin{cases}w & \text { in }\{w \geq \varepsilon\} \\ w^{\varepsilon} & \text { in } \overline{D_{R}^{\varepsilon}} \\ 0 & \text { otherwise. }\end{cases}
$$

Step II. Passage to the limit. If $(x, 0) \in \overline{D_{R}^{\varepsilon}}$, we have $0 \leq \frac{1}{\varepsilon} w(x, 0) \leq 1$. Since, from Lemma 4.1, we know that $0 \leq \psi^{+}(s) \leq 1$ for $s \geq 0$, it follows that $0 \leq w^{\varepsilon}(x, 0) \leq \varepsilon$. Applying the comparison principle for solutions of $P_{\varepsilon}$ we deduce that $0 \leq w^{\varepsilon} \leq \varepsilon$. Hence,

$$
\sup _{\bar{Q}}\left|v^{\varepsilon}-w\right| \leq 2 \varepsilon
$$

and therefore, the convergence of the family $v^{\varepsilon}$ follows.

Step III. Let us show that there exists $\varepsilon_{0}>0$ such that the functions $v^{\varepsilon}$ are subsolutions to $P_{\varepsilon}$ for $\varepsilon<\varepsilon_{0}$.

If $v^{\varepsilon}>\varepsilon$, then $v^{\varepsilon}=w$, which by hypothesis is subcaloric. Since $\beta_{\varepsilon}(s)=0$ when $s>\varepsilon$, it follows that $v^{\varepsilon}$ are subsolutions to $P_{\varepsilon}$ here.

If $0<v^{\varepsilon}<\varepsilon$, then we are in $D_{R}^{\varepsilon}$ and therefore, by construction, $v^{\varepsilon}$ are solutions to $P_{\varepsilon}$

If $v^{\varepsilon} \equiv 0$, the same conclusion holds, due to the fact that $\beta_{\varepsilon}(0)=0$.

That is, the $v^{\varepsilon}$ 's are continuous functions, and they are piecewise subsolutions to $P_{\varepsilon}$. In order to see that $v^{\varepsilon}$ are globally subsolutions to $P_{\varepsilon}$, it suffices to see that the jumps of the gradients (which occur at smooth surfaces), have the right sign.

To this effect, we will show that there exists $\varepsilon_{0}>0$ such that

$$
\left|\nabla w^{\varepsilon}\right| \leq \sqrt{2 M+\delta_{0} / 2} \text { on }\{w=\varepsilon\} \text {, for } \varepsilon<\varepsilon_{0} .
$$

Assume that (5.1) does not hold. Then, for every $j \in \mathbb{N}$, there exist $\varepsilon_{j}>0$ and $\left(x_{\varepsilon_{j}}, t_{\varepsilon_{j}}\right) \in Q$, with

$$
\varepsilon_{j} \rightarrow 0 \quad \text { and } \quad\left(x_{\varepsilon_{j}}, t_{\varepsilon_{j}}\right) \rightarrow\left(x_{0}, t_{0}\right) \in\{w=0\} \cap \partial\{w>0\},
$$

such that

$$
w^{\varepsilon_{j}}\left(x_{\varepsilon_{j}}, t_{\varepsilon_{j}}\right)=\varepsilon_{j} \quad \text { and } \quad\left|\nabla w^{\varepsilon_{j}}\left(x_{\varepsilon_{j}}, t_{\varepsilon_{j}}\right)\right|>\sqrt{2 M+\delta_{0} / 2} .
$$

From now on we will drop the subscript $j$ when referring to the sequences defined above and $\varepsilon \rightarrow 0$ will mean $j \rightarrow \infty$.

Since on the lateral boundary $\partial_{N} D_{R}^{\varepsilon}$ we have the Neumann data $\frac{\partial w^{\varepsilon}}{\partial \eta}=0$, we will use a reflection argument and assume that the points $\left(x_{\varepsilon}, t_{\varepsilon}\right)$ are far from the lateral boundary (with a different equation).

In fact, if $\left(x_{0}, t_{0}\right) \in \mathbb{R} \times \partial \Sigma \times[0, T]($ or $(0,+\infty) \times \partial \Sigma \times[0, T])$, we apply Proposition A.1 in the Appendix and deduce that there exists a change of variables $y=h(x)$ such that $h\left(x_{0}\right)=0$ and such that the function

$$
u^{\varepsilon}(y, t)=\left\{\begin{array}{l}
w^{\varepsilon}(x, t) \quad \text { for } y_{N} \geq 0, \\
u^{\varepsilon}\left(y_{1}, \cdots, y_{N-1},-y_{N}, t\right) \text { for } y_{N}<0
\end{array}\right.
$$

is a weak solution to

$$
\sum_{i, j} \frac{\partial}{\partial y_{i}}\left(a_{i j}(y) \frac{\partial u^{\varepsilon}}{\partial y_{j}}\right)+\sum_{i} b_{i}(y, t) \frac{\partial u^{\varepsilon}}{\partial y_{i}}-u_{t}^{\varepsilon}=\beta_{\varepsilon}\left(u^{\varepsilon}\right) \text { in }\left\{u^{\varepsilon}<\varepsilon\right\}
$$

for $y$ in a neighborhood $\mathcal{N}$ of the origin and $t \in[0, T]$. Here $a_{i j} \in W^{1, \infty}(\mathcal{N})$, $b_{i} \in L^{\infty}(\mathcal{N} \times[0, T])$. 
We choose the variables in such a way that $\nabla h_{1}\left(x_{0}\right)=-\frac{\nabla w^{+}\left(x_{0}, t_{0}\right)}{\left|\nabla w^{+}\left(x_{0}, t_{0}\right)\right|}, \nabla h_{i}\left(x_{0}\right)$. $\nabla h_{j}\left(x_{0}\right)=\delta_{i j}$ and $a_{i j}(0)=\delta_{i j}$.

We will sometimes denote $y=\left(y_{1}, y^{\prime}\right)$. And we denote $y_{\varepsilon}=h\left(x_{\varepsilon}\right)$.

We point out that the change of variables, the neighborhood $\mathcal{N}$ and the coefficients $a_{i j}$ in the equation depend only on the domain $\Sigma$; the coefficients $b_{i}$ depend only on $\Sigma$ and the coefficients $a_{i}$. In particular, all of them are independent of $F=\varepsilon-w$ in Proposition A.1.

If, on the other hand, $\left(x_{0}, t_{0}\right) \in \Omega \times[0, T]$ we change the origin and perform a rotation in the space variables and we are in a situation similar to the one above, with $u^{\varepsilon}(y, t)=w^{\varepsilon}(x, t)$ for $y \in \mathcal{N}$.

In any case, since $\nabla h_{1}\left(x_{0}\right)=-\frac{\nabla w^{+}\left(x_{0}, t_{0}\right)}{\left|\nabla w^{+}\left(x_{0}, t_{0}\right)\right|}, \nabla h_{i}\left(x_{0}\right) \cdot \nabla h_{j}\left(x_{0}\right)=\delta_{i j}$ and $\left\{w^{\varepsilon}=\right.$ $\varepsilon\}=\{w=\varepsilon\}$, there exists a family $f_{\varepsilon}$ of smooth functions such that, in a neighborhood of $\left(y_{\varepsilon}, t_{\varepsilon}\right)$,

$$
\begin{aligned}
& \left\{u^{\varepsilon}=\varepsilon\right\}=\left\{(y, t) / y_{1}-y_{\varepsilon 1}=f_{\varepsilon}\left(y^{\prime}-y_{\varepsilon}{ }^{\prime}, t-t_{\varepsilon}\right)\right\}, \\
& \left\{u^{\varepsilon}<\varepsilon\right\}=\left\{(y, t) / y_{1}-y_{\varepsilon 1}>f_{\varepsilon}\left(y^{\prime}-y_{\varepsilon}{ }^{\prime}, t-t_{\varepsilon}\right)\right\},
\end{aligned}
$$

where there holds that

$$
f_{\varepsilon}(0,0)=0, \quad\left|\nabla_{y^{\prime}} f_{\varepsilon}(0,0)\right| \rightarrow 0, \quad \varepsilon \rightarrow 0 .
$$

We can assume that (5.3) holds in $\left(B_{\rho}\left(y_{\varepsilon}\right) \times\left(t_{\varepsilon}-\rho^{2}, t_{\varepsilon}+\rho^{2}\right)\right) \cap\{0 \leq t \leq T\}$ for some $\rho>0$.

Let us now define

$$
\begin{aligned}
& \bar{u}^{\varepsilon}(y, t)=\frac{1}{\varepsilon} u^{\varepsilon}\left(y_{\varepsilon}+\varepsilon y, t_{\varepsilon}+\varepsilon^{2} t\right), \\
& \bar{f}_{\varepsilon}\left(y^{\prime}, t\right)=\frac{1}{\varepsilon} f_{\varepsilon}\left(\varepsilon y^{\prime}, \varepsilon^{2} t\right),
\end{aligned}
$$

and let

$$
\tau_{\varepsilon}=\frac{t_{\varepsilon}}{\varepsilon^{2}}, \gamma_{\varepsilon}=\frac{T-t_{\varepsilon}}{\varepsilon^{2}} .
$$

We have, for a subsequence,

$$
\tau_{\varepsilon} \rightarrow \tau, \gamma_{\varepsilon} \rightarrow \gamma
$$

where $0 \leq \tau, \gamma \leq+\infty$ and $\tau$ and $\gamma$ cannot be both finite.

We now let

$$
\mathcal{A}_{\varepsilon}=\left\{(y, t) /|y|<\frac{\rho}{\varepsilon},-\min \left(\tau_{\varepsilon}, \frac{\rho^{2}}{\varepsilon^{2}}\right)<t<\min \left(\gamma_{\varepsilon}, \frac{\rho^{2}}{\varepsilon^{2}}\right)\right\} .
$$

Then, the functions $\bar{u}^{\varepsilon}$ are weak solutions to

$$
\begin{aligned}
& \sum_{i, j} \frac{\partial}{\partial y_{i}}\left(a_{i j}^{\varepsilon}(y) \frac{\partial \bar{u}^{\varepsilon}}{\partial y_{j}}\right)+ \sum_{i} b_{i}^{\varepsilon}(y, t) \frac{\partial \bar{u}^{\varepsilon}}{\partial y_{i}}-\bar{u}_{t}^{\varepsilon}=\beta\left(\bar{u}^{\varepsilon}\right) \quad \text { in }\left\{y_{1}>\bar{f}_{\varepsilon}\left(y^{\prime}, t\right)\right\} \cap \mathcal{A}_{\varepsilon} \\
& \bar{u}^{\varepsilon}=1 \quad \text { on }\left\{y_{1}=\bar{f}_{\varepsilon}\left(y^{\prime}, t\right)\right\} \cap \mathcal{A}_{\varepsilon} \\
& 0 \leq \bar{u}^{\varepsilon} \leq 1 \quad \text { in }\left\{y_{1} \geq \bar{f}_{\varepsilon}\left(y^{\prime}, t\right)\right\} \cap \overline{\mathcal{A}_{\varepsilon}}
\end{aligned}
$$

where $a_{i j}^{\varepsilon}(y)=a_{i j}\left(y_{\varepsilon}+\varepsilon y\right), b_{i}^{\varepsilon}(y, t)=\varepsilon b_{i}\left(y_{\varepsilon}+\varepsilon y, t_{\varepsilon}+\varepsilon^{2} t\right)$. Note that we are under the hypotheses of Lemma 4.4. Then, there exists a function $\bar{u}$ such that, for 
a subsequence,

$$
\begin{aligned}
\bar{u} & \in C^{2+\alpha, 1+\frac{\alpha}{2}}\left(\left\{y_{1} \geq 0,-\tau<t<\gamma\right\}\right), \\
\bar{u}^{\varepsilon} & \rightarrow \bar{u} \quad \text { uniformly on compact subsets of }\left\{y_{1}>0,-\tau<t<\gamma\right\}, \\
\Delta \bar{u}-\bar{u}_{t}=\beta(\bar{u}) \quad \text { in }\left\{y_{1}>0,-\tau<t<\gamma\right\}, & \text { on }\left\{y_{1}=0,-\tau<t<\gamma\right\} \\
\bar{u}=1 & \text { in }\left\{y_{1} \geq 0,-\tau<t<\gamma\right\} .
\end{aligned}
$$

We will divide the remainder of the proof into two cases, depending on whether $\tau=+\infty$ or $\tau<+\infty$.

Case I. Assume $\tau=+\infty$.

In this case, Lemma 4.4 also gives

$$
\left|\nabla \bar{u}^{\varepsilon}(0,0)\right| \rightarrow|\nabla \bar{u}(0,0)| .
$$

On the other hand, $\bar{u}$ satisfies the hypotheses of Lemma 4.3 and therefore,

$$
|\nabla \bar{u}| \leq \sqrt{2 M} \text { on }\left\{y_{1}=0\right\}
$$

which yields

$$
\left|\nabla \bar{u}^{\varepsilon}(0,0)\right| \leq \sqrt{2 M+\delta_{0} / 4}
$$

for $\varepsilon$ small. But this gives

$$
\left|\nabla w^{\varepsilon}\left(x_{\varepsilon}, t_{\varepsilon}\right)\right| \leq \sqrt{2 M+\delta_{0} / 2},
$$

for $\varepsilon$ small. This contradicts (5.2) and completes the proof in case $\tau=+\infty$.

Case II. Assume $\tau<+\infty$. (In this case $\gamma=+\infty$.)

There holds that $\bar{u}^{\varepsilon}\left(y,-\tau_{\varepsilon}\right)=\frac{1}{\varepsilon} u^{\varepsilon}\left(y_{\varepsilon}+\varepsilon y, 0\right)$, then

$$
\bar{u}^{\varepsilon}\left(y,-\tau_{\varepsilon}\right)=\varphi\left(\frac{1}{\varepsilon} w\left(h^{-1}\left(y_{\varepsilon}+\varepsilon y\right), 0\right)\right) \chi\left(\tilde{w}\left(h^{-1}\left(y_{\varepsilon}+\varepsilon y\right)\right)\right)
$$

when $x_{0} \in \mathbb{R} \times \Sigma$. When $x_{0} \in \mathbb{R} \times \partial \Sigma$, (5.4) holds for $\left(y_{\varepsilon}+\varepsilon y\right)_{N} \geq 0$ and we obtain $\bar{u}^{\varepsilon}\left(y,-\tau_{\varepsilon}\right)$ for $\left(y_{\varepsilon}+\varepsilon y\right)_{N}<0$, recalling that

$$
u^{\varepsilon}(y, 0)=u^{\varepsilon}\left(y_{1}, \cdots, y_{N-1},-y_{N}, 0\right) \quad \text { for } y_{N}<0 .
$$

We want to apply here the result of Lemma 4.4 corresponding to $\tau<+\infty$. In fact, we can see that there exist $C, r>0$ such that $\left\|\bar{u}^{\varepsilon}\left(y,-\tau_{\varepsilon}\right)\right\|_{C^{1+\alpha}\left(\bar{B}_{r}(0)\right)} \leq C$. In case $x_{0} \in \mathbb{R} \times \Sigma$ we use the fact that $w$ is locally Lipschitz continuous in $\bar{\Omega} \times[0, T]$ and $\frac{t_{\varepsilon}}{\varepsilon} \rightarrow 0$ (and therefore, $t_{0}=0$ ) when $\tau<+\infty$, so that $w\left(h^{-1}\left(y_{\varepsilon}+\varepsilon y\right), 0\right) \geq \frac{\varepsilon}{2}$ for $|y|<r_{1}$ and $\varepsilon$ small. We also use that $\varphi \in C^{2}(1-m,+\infty)$ for some $m>0$. In case that $x_{0} \in \mathbb{R} \times \partial \Sigma$ we argue in a similar way and we also use (see Proposition A.1) that $\frac{\partial u^{\varepsilon}}{\partial y_{N}}(y, 0)=0$ on $\left\{y_{N}=0\right\}$ if $\varepsilon$ is small and $y$ bounded.

Now Lemma 4.4 gives, for a subsequence,

$$
\begin{gathered}
\bar{u} \in C^{\alpha, \frac{\alpha}{2}}\left(\left\{y_{1} \geq 0, t \geq-\tau\right\}\right), \\
\bar{u}^{\varepsilon}\left(y,-\tau_{\varepsilon}\right) \rightarrow \bar{u}(y,-\tau) \quad \text { uniformly on compact subsets of }\left\{y_{1}>0\right\} .
\end{gathered}
$$

Now using that $\nabla h_{1}\left(x_{0}\right)=-\frac{\nabla w^{+}\left(x_{0}, t_{0}\right)}{\left|\nabla w^{+}\left(x_{0}, t_{0}\right)\right|}$ and $\nabla h_{1}\left(x_{0}\right) \cdot \nabla h_{i}\left(x_{0}\right)=\delta_{i 1}$, we get that

$$
\bar{u}(y,-\tau)=\varphi\left(1-\left|\nabla w^{+}\left(x_{0}, t_{0}\right)\right| y_{1}\right) .
$$


We know by hypothesis that $\left|\nabla w^{+}\left(x_{0}, t_{0}\right)\right|=\sqrt{2 M+\delta_{0}}$, thus,

$$
\bar{u}(y,-\tau)=\psi^{+}\left(y_{1}\right) .
$$

(Notice that, in particular, the function $\bar{u}$ depends only on $y_{1}$ and $t$.)

Since the function $\psi^{+}\left(y_{1}\right)$ is a stationary subsolution to equation $P_{1}$, bounded for $y_{1} \geq 0$, and $\bar{u}=\psi^{+}$on the parabolic boundary of the domain $\left\{y_{1}>0, t>-\tau\right\}$, we conclude that

$$
\bar{u}(y, t) \geq \psi^{+}\left(y_{1}\right) \quad \text { in }\left\{y_{1} \geq 0, t \geq-\tau\right\} .
$$

It follows that

$$
|\nabla \bar{u}| \leq \sqrt{2 M+\delta_{0} / 4} \quad \text { on }\left\{y_{1}=0, t \geq-\tau\right\} .
$$

But Lemma 4.4 gives

$$
\left|\nabla \bar{u}^{\varepsilon}(0,0)\right| \rightarrow|\nabla \bar{u}(0,0)|
$$

which yields

$$
\left|\nabla \bar{u}^{\varepsilon}(0,0)\right| \leq \sqrt{2 M+3 \delta_{0} / 8}
$$

for $\varepsilon$ small. Then,

$$
\left|\nabla w^{\varepsilon}\left(x_{\varepsilon}, t_{\varepsilon}\right)\right| \leq \sqrt{2 M+\delta_{0} / 2}
$$

for $\varepsilon$ small. This contradicts (5.2) and completes the proof in case $\tau<+\infty$.

Theorem 5.2. Let $w$ be a classical supersolution to $P$ in $Q$, with $\frac{\partial w}{\partial \eta}=0$ on $\partial_{N} Q$, satisfying (H1). Assume, in addition, that there exists $\delta_{0}>0$ such that

$$
\left|\nabla w^{+}\right|=\sqrt{2 M-\delta_{0}} \quad \text { on } Q \cap \partial\{w>0\} .
$$

Then, there exists a family $v^{\varepsilon} \in C(\bar{Q})$, with $\nabla v^{\varepsilon} \in L_{\text {loc }}^{2}(\bar{Q})$, of weak supersolutions to $P_{\varepsilon}$ in $Q$, with $\frac{\partial v^{\varepsilon}}{\partial \eta}=0$ on $\partial_{N} Q$, such that, as $\varepsilon \rightarrow 0, v^{\varepsilon} \rightarrow w$ uniformly in $\bar{Q}$.

Moreover, there exists $0<\delta<1$ such that $v^{\varepsilon}=w$ in $\{w \geq(1-\delta) \varepsilon\}$ and $\nabla v^{\varepsilon} \in C(\bar{Q} \cap\{w \leq(1-\delta) \varepsilon\} \cap\{t>0\})$.

Proof. Step I. Construction of the family $v^{\varepsilon}$. Let $0<\delta<1$ be such that

$$
B(1-\delta)=\int_{0}^{1-\delta} \beta(\rho) d \rho=M-\delta_{0} / 8
$$

For every $\varepsilon>0$ small, we define the domain $D^{\varepsilon} \subset Q$ in the following way:

$$
D^{\varepsilon}=\left\{(x, t) \in Q / x_{1}>g_{(1-\delta) \varepsilon}\left(x^{\prime}, t\right)\right\} \text {. }
$$

Let $w^{\varepsilon}$ be the bounded solution to $P_{\varepsilon}$ in $D^{\varepsilon}$ with boundary data

$$
\begin{aligned}
w^{\varepsilon}(x, t) & = \begin{cases}(1-\delta) \varepsilon & \text { on } x_{1}=g_{(1-\delta) \varepsilon}\left(x^{\prime}, t\right), \\
w_{0}^{\varepsilon}(x) & \text { on } \partial D^{\varepsilon} \cap\{t=0\},\end{cases} \\
\frac{\partial w^{\varepsilon}}{\partial \eta} & =0 \text { on } \partial_{N} D^{\varepsilon}:=\partial_{N} Q \cap\left\{x_{1}>g_{(1-\delta) \varepsilon}\left(x^{\prime}, t\right)\right\} .
\end{aligned}
$$

In order to give the initial data $w_{0}^{\varepsilon}$, we let $\psi=\psi_{a, b}$ be the solution to (4.1) with

$$
a=1-\delta, \quad b=M-\delta_{0} / 8 .
$$

We now let

$$
\varphi(\xi)=\psi\left(\frac{1-\delta-\xi}{\sqrt{2 M-\delta_{0}}}\right)
$$


and we define

$$
w_{0}^{\varepsilon}(x)=\varepsilon \varphi\left(\frac{1}{\varepsilon} w(x, 0)\right) .
$$

For the existence and regularity of such a solution we refer to [LVW], Theorem 2.1, where it is shown that there exists a unique solution $w^{\varepsilon} \in L^{\infty}\left(D^{\varepsilon}\right) \cap C\left(\overline{D^{\varepsilon}}\right)$, with $\nabla w^{\varepsilon} \in C\left(\overline{D^{\varepsilon}} \cap\{t>0\}\right) \cap L_{\text {loc }}^{2}\left(\overline{D^{\varepsilon}}\right)$.

Finally, we define the family $v^{\varepsilon}$ as follows:

$$
v^{\varepsilon}= \begin{cases}w & \text { in }\{w \geq(1-\delta) \varepsilon\} \\ w^{\varepsilon} & \text { in } \overline{D^{\varepsilon}}\end{cases}
$$

Step II. Passage to the limit. If $(x, 0) \in \overline{D^{\varepsilon}}$, we have $0 \leq \frac{1}{\varepsilon} w(x, 0) \leq 1-\delta$. Since, from Lemma 4.1, we know that $0 \leq \psi(s) \leq 1-\delta$ for $s \geq 0$, it follows that $0 \leq w^{\varepsilon}(x, 0) \leq(1-\delta) \varepsilon$. Since $\beta_{\varepsilon}(s) \geq 0$, constant functions are supersolutions to $P_{\varepsilon}$. Therefore the comparison principle for bounded super and subsolutions of $P_{\varepsilon}$ implies that $0 \leq w^{\varepsilon} \leq(1-\delta) \varepsilon$. Hence,

$$
\sup _{\bar{Q}}\left|v^{\varepsilon}-w\right| \leq 2(1-\delta) \varepsilon
$$

and therefore, the convergence of the family $v^{\varepsilon}$ follows.

Step III. Let us show that there exists $\varepsilon_{0}>0$ such that the functions $v^{\varepsilon}$ are supersolutions to $P_{\varepsilon}$ for $\varepsilon<\varepsilon_{0}$.

If $v^{\varepsilon}>(1-\delta) \varepsilon$, then $v^{\varepsilon}=w$, which by hypothesis is supercaloric. Since $\beta_{\varepsilon}(s) \geq 0$, it follows that $v^{\varepsilon}$ are supersolutions to $P_{\varepsilon}$ here.

If $v^{\varepsilon}<(1-\delta) \varepsilon$, then we are in $D^{\varepsilon}$ and therefore, by construction, $v^{\varepsilon}$ are solutions to $P_{\varepsilon}$.

That is, the $v^{\varepsilon}$ 's are continuous functions, and they are piecewise supersolutions to $P_{\varepsilon}$. In order to see that $v^{\varepsilon}$ are globally supersolutions to $P_{\varepsilon}$, it suffices to see that the jumps of the gradients (which occur at smooth surfaces), have the right sign.

To this effect, we will show that there exists $\varepsilon_{0}>0$ such that

$$
\left|\nabla w^{\varepsilon}\right| \geq \sqrt{2 M-\delta_{0} / 2} \quad \text { on }\{w=(1-\delta) \varepsilon\}, \text { for } \varepsilon<\varepsilon_{0} .
$$

Assume that (5.5) does not hold. Then, for every $j \in \mathbb{N}$, there exist $\varepsilon_{j}>0$ and $\left(x_{\varepsilon_{j}}, t_{\varepsilon_{j}}\right) \in Q$, with

$$
\varepsilon_{j} \rightarrow 0 \quad \text { and } \quad\left(x_{\varepsilon_{j}}, t_{\varepsilon_{j}}\right) \rightarrow\left(x_{0}, t_{0}\right) \in\{w=0\} \cap \partial\{w>0\}
$$

such that

$$
w^{\varepsilon_{j}}\left(x_{\varepsilon_{j}}, t_{\varepsilon_{j}}\right)=(1-\delta) \varepsilon_{j} \quad \text { and } \quad\left|\nabla w^{\varepsilon_{j}}\left(x_{\varepsilon_{j}}, t_{\varepsilon_{j}}\right)\right|<\sqrt{2 M-\delta_{0} / 2} .
$$

From now on we will drop the subscript $j$ when referring to the sequences defined above and $\varepsilon \rightarrow 0$ will mean $j \rightarrow \infty$.

Since on the lateral boundary $\partial_{N} D^{\varepsilon}$, we have the Neumann data $\frac{\partial w^{\varepsilon}}{\partial \eta}=0$, we will use a reflection argument and assume that the points $\left(x_{\varepsilon}, t_{\varepsilon}\right)$ are far from the lateral boundary (with a different equation). To this effect we will proceed exactly as in Theorem 5.1.

In fact, if $\left(x_{0}, t_{0}\right) \in \mathbb{R} \times \partial \Sigma \times[0, T]($ or $(0,+\infty) \times \partial \Sigma \times[0, T])$, we apply Proposition A.1 in the Appendix and deduce that there exists a change of variables $y=h(x)$ 
such that $h\left(x_{0}\right)=0$ and such that the function

$$
u^{\varepsilon}(y, t)=\left\{\begin{array}{l}
w^{\varepsilon}(x, t) \quad \text { for } y_{N} \geq 0, \\
u^{\varepsilon}\left(y_{1}, \cdots, y_{N-1},-y_{N}, t\right) \quad \text { for } y_{N}<0
\end{array}\right.
$$

is a weak solution to

$$
\sum_{i, j} \frac{\partial}{\partial y_{i}}\left(a_{i j}(y) \frac{\partial u^{\varepsilon}}{\partial y_{j}}\right)+\sum_{i} b_{i}(y, t) \frac{\partial u^{\varepsilon}}{\partial y_{i}}-u_{t}^{\varepsilon}=\beta_{\varepsilon}\left(u^{\varepsilon}\right) \text { in }\left\{u^{\varepsilon}<(1-\delta) \varepsilon\right\}
$$

for $y$ in a neighborhood $\mathcal{N}$ of the origin and $t \in[0, T]$. Here $a_{i j} \in W^{1, \infty}(\mathcal{N})$, $b_{i} \in L^{\infty}(\mathcal{N} \times[0, T])$.

We choose the variables in such a way that $\nabla h_{1}\left(x_{0}\right)=-\frac{\nabla w^{+}\left(x_{0}, t_{0}\right)}{\left|\nabla w^{+}\left(x_{0}, t_{0}\right)\right|}, \nabla h_{i}\left(x_{0}\right)$. $\nabla h_{j}\left(x_{0}\right)=\delta_{i j}$ and $a_{i j}(0)=\delta_{i j}$.

We will sometimes denote $y=\left(y_{1}, y^{\prime}\right)$. And we denote $y_{\varepsilon}=h\left(x_{\varepsilon}\right)$.

We point out that the change of variables, the neighborhood $\mathcal{N}$ and the coefficients $a_{i j}$ in the equation depend only on the domain $\Sigma$; the coefficients $b_{i}$ depend only on $\Sigma$ and the coefficients $a_{i}$. In particular, all of them are independent of $F=(1-\delta) \varepsilon-w$ in Proposition A.1.

If, on the other hand, $\left(x_{0}, t_{0}\right) \in \Omega \times[0, T]$ we change the origin and perform a rotation in the space variables and we are in a situation similar to the one above, with $u^{\varepsilon}(y, t)=w^{\varepsilon}(x, t)$ for $y \in \mathcal{N}$.

In any case, since $\nabla h_{1}\left(x_{0}\right)=-\frac{\nabla w^{+}\left(x_{0}, t_{0}\right)}{\left|\nabla w^{+}\left(x_{0}, t_{0}\right)\right|}, \nabla h_{i}\left(x_{0}\right) \cdot \nabla h_{j}\left(x_{0}\right)=\delta_{i j}$ and $\left\{w^{\varepsilon}=\right.$ $(1-\delta) \varepsilon\}=\{w=(1-\delta) \varepsilon\}$, it follows that there exists a family $f_{\varepsilon}$ of smooth functions such that, in a neighborhood of $\left(y_{\varepsilon}, t_{\varepsilon}\right)$,

$$
\begin{aligned}
& \left\{u^{\varepsilon}=(1-\delta) \varepsilon\right\}=\left\{(y, t) / y_{1}-y_{\varepsilon 1}=f_{\varepsilon}\left(y^{\prime}-y_{\varepsilon}{ }^{\prime}, t-t_{\varepsilon}\right)\right\}, \\
& \left\{u^{\varepsilon}<(1-\delta) \varepsilon\right\}=\left\{(y, t) / y_{1}-y_{\varepsilon_{1}}>f_{\varepsilon}\left(y^{\prime}-y_{\varepsilon}{ }^{\prime}, t-t_{\varepsilon}\right)\right\},
\end{aligned}
$$

where there holds that

$$
f_{\varepsilon}(0,0)=0, \quad\left|\nabla_{y^{\prime}} f_{\varepsilon}(0,0)\right| \rightarrow 0, \quad \varepsilon \rightarrow 0 .
$$

We can assume that (5.7) holds in $\left(B_{\rho}\left(y_{\varepsilon}\right) \times\left(t_{\varepsilon}-\rho^{2}, t_{\varepsilon}+\rho^{2}\right)\right) \cap\{0 \leq t \leq T\}$ for some $\rho>0$.

Let us now define

$$
\begin{aligned}
& \bar{u}^{\varepsilon}(y, t)=\frac{1}{\varepsilon} u^{\varepsilon}\left(y_{\varepsilon}+\varepsilon y, t_{\varepsilon}+\varepsilon^{2} t\right), \\
& \bar{f}_{\varepsilon}\left(y^{\prime}, t\right)=\frac{1}{\varepsilon} f_{\varepsilon}\left(\varepsilon y^{\prime}, \varepsilon^{2} t\right),
\end{aligned}
$$

and let

$$
\tau_{\varepsilon}=\frac{t_{\varepsilon}}{\varepsilon^{2}}, \gamma_{\varepsilon}=\frac{T-t_{\varepsilon}}{\varepsilon^{2}}
$$

We have, for a subsequence,

$$
\tau_{\varepsilon} \rightarrow \tau, \gamma_{\varepsilon} \rightarrow \gamma
$$

where $0 \leq \tau, \gamma \leq+\infty$ and $\tau$ and $\gamma$ cannot be both finite.

We now let

$$
\mathcal{A}_{\varepsilon}=\left\{(y, t) /|y|<\frac{\rho}{\varepsilon},-\min \left(\tau_{\varepsilon}, \frac{\rho^{2}}{\varepsilon^{2}}\right)<t<\min \left(\gamma_{\varepsilon}, \frac{\rho^{2}}{\varepsilon^{2}}\right)\right\} .
$$


Then, the functions $\bar{u}^{\varepsilon}$ are weak solutions to

$$
\begin{gathered}
\sum_{i, j} \frac{\partial}{\partial y_{i}}\left(a_{i j}^{\varepsilon}(y) \frac{\partial \bar{u}^{\varepsilon}}{\partial y_{j}}\right)+\sum_{i} b_{i}^{\varepsilon}(y, t) \frac{\partial \bar{u}^{\varepsilon}}{\partial y_{i}}-\bar{u}_{t}^{\varepsilon}=\beta\left(\bar{u}^{\varepsilon}\right) \quad \text { in }\left\{y_{1}>\bar{f}_{\varepsilon}\left(y^{\prime}, t\right)\right\} \cap \mathcal{A}_{\varepsilon} \\
\bar{u}^{\varepsilon}=1-\delta \quad \text { on }\left\{y_{1}=\bar{f}_{\varepsilon}\left(y^{\prime}, t\right)\right\} \cap \mathcal{A}_{\varepsilon} \\
0 \leq \bar{u}^{\varepsilon} \leq 1-\delta \quad \text { in }\left\{y_{1} \geq \bar{f}_{\varepsilon}\left(y^{\prime}, t\right)\right\} \cap \overline{\mathcal{A}_{\varepsilon}}
\end{gathered}
$$

where $a_{i j}^{\varepsilon}(y)=a_{i j}\left(y_{\varepsilon}+\varepsilon y\right), b_{i}^{\varepsilon}(y, t)=\varepsilon b_{i}\left(y_{\varepsilon}+\varepsilon y, t_{\varepsilon}+\varepsilon^{2} t\right)$. Note that we are under the hypotheses of Lemma 4.4. Then, there exists a function $\bar{u}$ such that, for a subsequence,

$$
\begin{aligned}
& \bar{u} \in C^{2+\alpha, 1+\frac{\alpha}{2}}\left(\left\{y_{1} \geq 0,-\tau<t<\gamma\right\}\right), \\
& \bar{u}^{\varepsilon} \rightarrow \bar{u} \quad \text { uniformly on compact subsets of }\left\{y_{1}>0,-\tau<t<\gamma\right\}, \\
& \Delta \bar{u}-\bar{u}_{t}=\beta(\bar{u}) \quad \text { in }\left\{y_{1}>0,-\tau<t<\gamma\right\}, \\
& \bar{u}=1-\delta \quad \text { on }\left\{y_{1}=0,-\tau<t<\gamma\right\}, \\
& 0 \leq \bar{u} \leq 1-\delta \quad \text { in }\left\{y_{1} \geq 0,-\tau<t<\gamma\right\} .
\end{aligned}
$$

We will divide the remainder of the proof into two cases, depending on whether $\tau=+\infty$ or $\tau<+\infty$.

Case I. Assume $\tau=+\infty$.

In this case, Lemma 4.4 also gives

$$
\left|\nabla \bar{u}^{\varepsilon}(0,0)\right| \rightarrow|\nabla \bar{u}(0,0)|
$$

On the other hand, $\bar{u}$ satisfies the hypotheses of Lemma 4.3 and therefore,

$$
|\nabla \bar{u}|=\sqrt{2 M-\delta_{0} / 4} \text { on }\left\{y_{1}=0\right\}
$$

which yields

$$
\left|\nabla \bar{u}^{\varepsilon}(0,0)\right| \geq \sqrt{2 M-3 \delta_{0} / 8}
$$

for $\varepsilon$ small. But this gives

$$
\left|\nabla w^{\varepsilon}\left(x_{\varepsilon}, t_{\varepsilon}\right)\right| \geq \sqrt{2 M-\delta_{0} / 2},
$$

for $\varepsilon$ small. This contradicts (5.6) and completes the proof in case $\tau=+\infty$.

Case II. Assume $\tau<+\infty$. (In this case $\gamma=+\infty$.)

There holds that $\bar{u}^{\varepsilon}\left(y,-\tau_{\varepsilon}\right)=\frac{1}{\varepsilon} u^{\varepsilon}\left(y_{\varepsilon}+\varepsilon y, 0\right)$, then

$$
\bar{u}^{\varepsilon}\left(y,-\tau_{\varepsilon}\right)=\varphi\left(\frac{1}{\varepsilon} w\left(h^{-1}\left(y_{\varepsilon}+\varepsilon y\right), 0\right)\right)
$$

when $x_{0} \in \mathbb{R} \times \Sigma$. When $x_{0} \in \mathbb{R} \times \partial \Sigma$, (5.8) holds for $\left(y_{\varepsilon}+\varepsilon y\right)_{N} \geq 0$ and we obtain $\bar{u}^{\varepsilon}\left(y,-\tau_{\varepsilon}\right)$ for $\left(y_{\varepsilon}+\varepsilon y\right)_{N}<0$, recalling that

$$
u^{\varepsilon}(y, 0)=u^{\varepsilon}\left(y_{1}, \cdots, y_{N-1},-y_{N}, 0\right) \text { for } y_{N}<0 .
$$

Here we want to apply the result of Lemma 4.4 corresponding to $\tau<+\infty$. In fact, we can see that there exist $C, r>0$ such that $\left\|\bar{u}^{\varepsilon}\left(y,-\tau_{\varepsilon}\right)\right\|_{C^{1+\alpha}\left(\bar{B}_{r}(0)\right)} \leq C$. In case $x_{0} \in \mathbb{R} \times \Sigma$ we use the fact that $w$ is locally Lipschitz continuous in $\bar{\Omega} \times[0, T]$ and $\frac{t_{\varepsilon}}{\varepsilon} \rightarrow 0$ (and therefore, $t_{0}=0$ ) when $\tau<+\infty$, so that $w\left(h^{-1}\left(y_{\varepsilon}+\varepsilon y\right), 0\right) \geq \frac{1}{2}(1-\delta) \varepsilon$ for $|y|<r_{1}$ and $\varepsilon$ small. We also use that $\varphi \in C^{2}$. In case that $x_{0} \in \mathbb{R} \times \partial \Sigma$ we argue in a similar way and we also use (see Proposition A.1) that $\frac{\partial u^{\varepsilon}}{\partial y_{N}}(y, 0)=0$ on $\left\{y_{N}=0\right\}$ if $\varepsilon$ is small and $y$ bounded. 
Now Lemma 4.4 gives, for a subsequence,

$$
\begin{gathered}
\bar{u} \in C^{\alpha, \frac{\alpha}{2}}\left(\left\{y_{1} \geq 0, t \geq-\tau\right\}\right), \\
\bar{u}^{\varepsilon}\left(y,-\tau_{\varepsilon}\right) \rightarrow \bar{u}(y,-\tau) \quad \text { uniformly on compact subsets of }\left\{y_{1}>0\right\} .
\end{gathered}
$$

Now using that $\nabla h_{1}\left(x_{0}\right)=-\frac{\nabla w^{+}\left(x_{0}, t_{0}\right)}{\left|\nabla w^{+}\left(x_{0}, t_{0}\right)\right|}$ and $\nabla h_{1}\left(x_{0}\right) \cdot \nabla h_{i}\left(x_{0}\right)=\delta_{i 1}$, we get that

$$
\bar{u}(y,-\tau)=\varphi\left(1-\delta-\left|\nabla w^{+}\left(x_{0}, t_{0}\right)\right| y_{1}\right) .
$$

We know, by hypothesis that $\left|\nabla w^{+}\left(x_{0}, t_{0}\right)\right|=\sqrt{2 M-\delta_{0}}$, thus,

$$
\bar{u}(y,-\tau)=\psi\left(y_{1}\right) .
$$

Since the function $\psi\left(y_{1}\right)$ is a stationary solution to equation $P_{1}$, bounded for $y_{1} \geq 0$, and $\bar{u}=\psi$ on the parabolic boundary of the domain $\left\{y_{1}>0, t>-\tau\right\}$, we conclude that

$$
\bar{u}(y, t)=\psi\left(y_{1}\right) \quad \text { in }\left\{y_{1} \geq 0, t \geq-\tau\right\} .
$$

It follows that

$$
|\nabla \bar{u}|=\sqrt{2 M-\delta_{0} / 4} \quad \text { on }\left\{y_{1}=0, t \geq-\tau\right\} .
$$

But Lemma 4.4 gives

$$
\left|\nabla \bar{u}^{\varepsilon}(0,0)\right| \rightarrow|\nabla \bar{u}(0,0)|,
$$

which yields

$$
\left|\nabla \bar{u}^{\varepsilon}(0,0)\right| \geq \sqrt{2 M-3 \delta_{0} / 8}
$$

for $\varepsilon$ small. Then,

$$
\left|\nabla w^{\varepsilon}\left(x_{\varepsilon}, t_{\varepsilon}\right)\right| \geq \sqrt{2 M-\delta_{0} / 2},
$$

for $\varepsilon$ small. This contradicts (5.6) and completes the proof in case $\tau<+\infty$.

\section{Existence And uniqueness of the Limit SOlution}

In this section we prove that, under certain assumptions, a classical solution to the initial and boundary value problem associated to $P$ is the uniform limit of any family of solutions to problem $P_{\varepsilon}$ with corresponding boundary data. This, in particular, implies that such limit exists and is unique. Moreover, it is independent of the choice of the function $\beta$.

In particular, under the assumptions of this section our classical solution is the unique classical solution and also the unique viscosity solution (by the results of Section 3).

First, we give the result in a semi-cylinder.

Theorem 6.1. Let $\Omega=(0,+\infty) \times \Sigma, Q=\Omega \times(0, T), \partial_{N} Q=(0, \infty) \times \partial \Sigma \times(0, T)$ and $\partial_{D} Q=\partial_{p} Q \backslash \partial_{N} Q$.

Let $u$ be a bounded classical solution to $P$ in $Q$, with $\frac{\partial u}{\partial \eta}=0$ on $\partial_{N} Q$, such that

$\left.u\right|_{\partial_{D} Q}$ has a bounded, nonempty free boundary and $u_{x_{1}}<0$ on $\overline{\{u>0\}} \cap \partial_{D} Q$.

Assume that $u\left(0, x^{\prime}, t\right)>0$ for $\left(x^{\prime}, t\right) \in \bar{\Sigma} \times[0, T]$ with $u\left(0, x^{\prime}, t\right) \in C^{2,1}(\bar{\Sigma} \times[0, T])$.

Let $u^{\varepsilon} \in C(\bar{Q})$ with $\nabla u^{\varepsilon} \in L_{l o c}^{2}(\bar{Q})$ be a family of bounded nonnegative weak solutions to $P_{\varepsilon}$ in $Q$, with $\frac{\partial u^{\varepsilon}}{\partial \eta}=0$ on $\partial_{N} Q$, such that $u^{\varepsilon} \rightarrow u$ uniformly on $\partial_{D} Q$ and $\left\{u^{\varepsilon}>0\right\} \cap \partial_{D} Q \rightarrow\{u>0\} \cap \partial_{D} Q$. Then $u^{\varepsilon} \rightarrow u$ uniformly in $\bar{Q}$. 
Remark 6.1. In order for $P_{\varepsilon}$ to approximate problem $P$ properly, we need to impose some condition on how the Dirichlet datum of $u$ is approximated by the Dirichlet datum of $u^{\varepsilon}$ so that we get a limit solution with a free boundary starting from the initial free boundary $\Omega \cap \partial\{x / u(x, 0)>0\}$. In fact, when $a_{i} \equiv 0$, if $u^{\varepsilon} \geq \varepsilon$ on $\partial_{D} Q$, then the limit function $\widehat{u}=\lim u^{\varepsilon}$ is the bounded solution to the heat equation with Dirichlet datum $u$. Therefore, the uniqueness result does not hold if we only require $u^{\varepsilon} \rightarrow u$ on $\partial_{D} Q$.

However, we can relax the assumption that $\left\{u^{\varepsilon}>0\right\} \cap \partial_{D} Q \rightarrow\{u>0\} \cap$ $\partial_{D} Q$, imposing instead a suitable control on the growth of $u^{\varepsilon}(x, 0)$ away from $\Omega \cap\{x / u(x, 0)>0\}$ and Theorem 6.1 still holds.

Proof of Theorem 6.1. Given $\rho>0$ and $\sigma>0$ small, we define in $\bar{Q}$

$$
u_{\rho, \sigma}(x, t)=(1+\sigma) u\left(x_{1}+\rho, x^{\prime}, t\right) .
$$

Then, $u_{\rho, \sigma}$ is a classical subsolution to $P$ in $Q$ with vanishing Neumann data on $\partial_{N} Q$. Given $\delta>0$ we choose $\rho, \sigma$ so that

$$
u_{\rho, \sigma} \geq u-\delta \quad \text { in } Q .
$$

On the other hand, using Proposition 2.5 and Corollary 3.3 we see that $u_{\rho, \sigma}$ satisfies the hypotheses of Theorem 5.1. Therefore, there exists a family $v^{\varepsilon}$ (depending on $\rho$ and $\sigma$ ) of weak subsolutions to $P_{\varepsilon}$ in $Q$, such that

$$
\begin{array}{cl}
\frac{\partial v^{\varepsilon}}{\partial \eta}=0 & \text { on } \partial_{N} Q, \\
v^{\varepsilon} \rightarrow u_{\rho, \sigma} & \text { uniformly in } \bar{Q}, \text { as } \varepsilon \rightarrow 0 .
\end{array}
$$

In addition, it follows from the construction of the family $v^{\varepsilon}$ that $\sigma$ can be chosen small enough (depending on $\rho$ ), so that we have

$$
\begin{aligned}
v^{\varepsilon} & \leq u^{\varepsilon} \quad \text { on } \partial_{D} Q, \\
\frac{\partial v^{\varepsilon}}{\partial \eta} & =\frac{\partial u^{\varepsilon}}{\partial \eta}=0 \quad \text { on } \partial_{N} Q .
\end{aligned}
$$

Consequently,

$$
v^{\varepsilon} \leq u^{\varepsilon} \quad \text { in } \bar{Q} \text {. }
$$

Therefore, there exists $\varepsilon_{0}(\delta)$ such that, if $\varepsilon \leq \varepsilon_{0}$,

$$
u_{\rho, \sigma}-\delta \leq u^{\varepsilon} \quad \text { in } \bar{Q}
$$

and finally we obtain

$$
u-2 \delta \leq u^{\varepsilon} \quad \text { in } \bar{Q} .
$$

In order to show that $u^{\varepsilon} \leq u+2 \delta$, we proceed in a similar way. But we first extend $u$ to a neighborhood of $x_{1}=0$ exactly as we did in Theorem 3.1. For example, in $-\mu \leq x_{1} \leq 0,\left(x^{\prime}, t\right) \in \bar{\Sigma} \times[0, T]$, we let

$$
u\left(x_{1}, x^{\prime}, t\right)=u\left(0, x^{\prime}, t\right)-c x_{1}-k x_{1}^{2},
$$

where $\mu, c$ and $k$ are positive constants, chosen as in Theorem 3.1 in such a way that $u>0$ in $-\mu \leq x_{1} \leq 0$ and $\mathcal{L} u \leq 0$ in $\left\{x_{1}>-\mu\right\} \cap\{u>0\}$.

For $0<\rho<\mu$ and $\sigma>0$ small, we define in $\bar{Q}$

$$
u^{\rho, \sigma}(x, t)=(1-\sigma) u\left(x_{1}-\rho, x^{\prime}, t\right) .
$$


We choose $\rho$ and $\sigma$ small enough so that

$$
u^{\rho, \sigma} \leq u+\delta \quad \text { in } Q .
$$

Now we fix $a>\rho$ small. Then, $u^{\rho, \sigma}$ is a classical supersolution to $P$ in $Q \cap\left\{x_{1}>a\right\}$ with vanishing Neumann data on $\partial_{N} Q \cap\left\{x_{1}>a\right\}$ which satisfies the hypotheses of Theorem 5.2 in the domain $Q \cap\left\{x_{1}>a\right\}$. Therefore, there exists a family $v^{\varepsilon}$ (depending on $\rho$ and $\sigma$ ) of weak supersolutions to $P_{\varepsilon}$ in $Q \cap\left\{x_{1}>a\right\}$, such that

$$
\begin{aligned}
\frac{\partial v^{\varepsilon}}{\partial \eta}=0 & \text { on } \partial_{N} Q \cap\left\{x_{1}>a\right\}, \\
v^{\varepsilon} \rightarrow u^{\rho, \sigma} & \text { uniformly in } \bar{Q} \cap\left\{x_{1} \geq a\right\}, \text { as } \varepsilon \rightarrow 0 .
\end{aligned}
$$

Notice that, by the construction in Theorem $5.2, v^{\varepsilon}=u^{\rho, \sigma}$ in a neighborhood of $x_{1}=a$. Then, if we extend $v^{\varepsilon}$ to the whole region $\bar{Q}$ letting

$$
v^{\varepsilon}=u^{\rho, \sigma} \quad \text { in } 0 \leq x_{1} \leq a,
$$

it follows that $v^{\varepsilon}$ are supersolutions to $P_{\varepsilon}$ in $Q$ satisfying (6.1) up to $x_{1}=0$.

We finally choose $\sigma$ small enough (depending on $\rho$ ) so that we have

$$
\begin{aligned}
v^{\varepsilon} & \geq u^{\varepsilon} \quad \text { on } \partial_{D} Q, \\
\frac{\partial v^{\varepsilon}}{\partial \eta} & =\frac{\partial u^{\varepsilon}}{\partial \eta}=0 \quad \text { on } \partial_{N} Q .
\end{aligned}
$$

It follows that

$$
v^{\varepsilon} \geq u^{\varepsilon} \quad \text { in } \bar{Q} .
$$

Therefore, there exists $\varepsilon_{1}(\delta)>0$ such that if $\varepsilon \leq \varepsilon_{1}$,

$$
u^{\rho, \sigma}+\delta \geq u^{\varepsilon} \quad \text { in } \bar{Q} .
$$

So that,

$$
u+2 \delta \geq u^{\varepsilon} \quad \text { in } \bar{Q} .
$$

Thus, $u^{\varepsilon}$ converges uniformly to $u$ in $\bar{Q}$.

A similar result holds for a full cylinder as spatial domain, under suitable monotonicity assumptions at $x_{1}=-\infty$.

Theorem 6.2. Let $\Omega=\mathbb{R} \times \Sigma, Q=\Omega \times(0, T), \partial_{N} Q=\mathbb{R} \times \partial \Sigma \times(0, T)$ and $\partial_{D} Q=\partial_{p} Q \backslash \partial_{N} Q$.

Let $u$ be a bounded classical solution to $P$ in $Q$, with $\frac{\partial u}{\partial \eta}=0$ on $\partial_{N} Q$ and $\|u\|_{C^{\alpha, \frac{\alpha}{2}}(\bar{Q})}<\infty$, such that $\left.u\right|_{\partial_{D} Q}$ has a bounded, nonempty free boundary.

Assume that $u_{x_{1}}<0$ on $\overline{\{u>0\}} \cap \partial_{D} Q$ and $u_{x_{1}}(x, 0) \leq-c_{1} e^{c_{2} x_{1}}$ for $x_{1} \leq-a$ for some constants $c_{1}, c_{2}, a>0$.

Let $u^{\varepsilon} \in C(\bar{Q})$ with $\nabla u^{\varepsilon} \in L_{\text {loc }}^{2}(\bar{Q})$ be a family of bounded nonnegative weak solutions to $P_{\varepsilon}$ in $Q$, with $\frac{\partial u^{\varepsilon}}{\partial \eta}=0$ on $\partial_{N} Q$, such that $u^{\varepsilon} \rightarrow u$ uniformly on $\partial_{D} Q$, with $\left\{u^{\varepsilon}>0\right\} \cap \partial_{D} Q \rightarrow\{u>0\} \cap \partial_{D} Q$ and $\left|u^{\varepsilon}(x, 0)-u(x, 0)\right| \leq k_{1} e^{-k_{2} x_{1}^{2}}$ for $x_{1} \leq-a$ for some constants $k_{1}, k_{2}>0$.

Then $u^{\varepsilon} \rightarrow u$ uniformly in $\bar{Q}$. 
Proof. Step I. Behavior of $u^{\varepsilon}$ and $u$ for $x_{1} \rightarrow-\infty$.

Let us first see that we can take $a$ large enough so that, for some $\varepsilon_{1}>0$,

$$
u^{\varepsilon} \geq \varepsilon \quad \text { in } x_{1} \leq-a \text {, if } \varepsilon \leq \varepsilon_{1} .
$$

In fact, let $c, K>0$ be such that, for $\varepsilon \leq \varepsilon_{0}$, there holds that $u^{\varepsilon}(x, 0) \geq 2 c$ for $x_{1} \leq-\frac{K}{2}$. For $\delta_{0}>0$ and $A \geq\left\|a_{1}\right\|_{L^{\infty}}$, let us consider the function

$$
v_{-}(x, t)=c\left(1-\exp \left\{\frac{\sqrt{2 M+\delta_{0}}}{c}\left(x_{1}+A t\right)+\frac{2 M+\delta_{0}}{c^{2}} t+\frac{K \sqrt{2 M+\delta_{0}}}{c}\right\}\right)^{+} .
$$

Then $v_{-}$is a bounded classical subsolution to $P$ in $Q$, with $\frac{\partial v_{-}}{\partial \eta}=0$ on $\partial_{N} Q$, such that $Q \cap \partial\left\{v_{-}>0\right\}$ is bounded and $\left|\nabla v_{-}\right|=\sqrt{2 M+\delta_{0}}$ on $Q \cap \partial\left\{v_{-}>0\right\}$. Moreover, there holds that $v_{-}(x, 0) \leq c$ for $x \in \Omega$ and $v_{-}(x, 0)=0$ for $x_{1} \geq-K$.

Since $v_{-}$satisfies the hypotheses of Theorem 5.1, we can construct a family $v_{-}^{\varepsilon}$ of weak subsolutions to $P_{\varepsilon}$ in $Q$, with $\frac{\partial v_{-}^{\varepsilon}}{\partial \eta}=0$ on $\partial_{N} Q$, such that $v_{-}^{\varepsilon} \rightarrow v_{-}$ uniformly in $\bar{Q}$ and $v_{-}^{\varepsilon}(x, 0)=0$ for $x_{1} \geq-\frac{K}{2}$. There exists $0<\varepsilon_{1} \leq \varepsilon_{0}$ such that $v_{-}^{\varepsilon}(x, 0) \leq 2 c$ if $\varepsilon \leq \varepsilon_{1}$ and thus,

$$
v_{-}^{\varepsilon}(x, 0) \leq u^{\varepsilon}(x, 0) \text { in } \Omega \text {, if } \varepsilon \leq \varepsilon_{1} .
$$

Therefore, $v_{-}^{\varepsilon} \leq u^{\varepsilon}$ in $\bar{Q}$. So that, if $a$ is taken large enough, (6.2) holds.

On the other hand, $u$ satisfies the hypotheses of Proposition 2.4. Therefore, $Q \cap \partial\{u>0\}$ is bounded. So that, we may choose $a$ large enough in order to have, in addition, that

$$
u>0 \quad \text { in } x_{1} \leq-a .
$$

Therefore, for $\varepsilon \leq \varepsilon_{1}$ and $w^{\varepsilon}=u^{\varepsilon}-u$, there holds that

$$
\begin{aligned}
& \mathcal{L} w^{\varepsilon}=0 \quad \text { in } x_{1}<-a, \quad 0<t<T, \\
& w^{\varepsilon}(x, 0) \leq k_{1} e^{-k_{2} x_{1}^{2}} \quad \text { in } x_{1}<-a, \\
& w^{\varepsilon} \leq L \quad \text { on } x_{1} \leq-a, \quad 0<t<T,
\end{aligned}
$$

for some constant $L$ independent of $\varepsilon$. Therefore, there exist $\bar{k}_{1}, \bar{k}_{2}>0$ such that, for some constant $l_{1}>a$ independent of $\varepsilon$,

$$
w^{\varepsilon}(x, t) \leq \bar{k}_{1} e^{-\bar{k}_{2} x_{1}^{2}} \quad \text { in } x_{1} \leq-l_{1} \text {, if } \varepsilon \leq \varepsilon_{1} .
$$

We may replace the function $w^{\varepsilon}$ above by $-w^{\varepsilon}$. Therefore,

$$
\left|u^{\varepsilon}(x, t)-u(x, t)\right| \leq \bar{k}_{1} e^{-\bar{k}_{2} x_{1}^{2}} \quad \text { in } x_{1} \leq-l_{1} \text {, if } \varepsilon \leq \varepsilon_{1} .
$$

Let us now analyze the behavior of $u_{x_{1}}$ for $x_{1} \rightarrow-\infty$. There holds that

$$
\begin{aligned}
& \mathcal{L} u_{x_{1}}=0 \quad \text { in } x_{1}<-a, \quad 0<t<T, \\
& u_{x_{1}}(x, 0) \leq-c_{1} e^{c_{2} x_{1}} \quad \text { in } x_{1}<-a, \\
& u_{x_{1}} \leq-r \quad \text { on } x_{1}=-a, \quad 0<t<T,
\end{aligned}
$$

for some positive constant $r$. Therefore, there exist $\bar{c}_{1}, \bar{c}_{2}>0$ and $l_{2}>a$ such that

$$
u_{x_{1}}(x, t) \leq-\bar{c}_{1} e^{\bar{c}_{2} x_{1}} \quad \text { in } x_{1} \leq-l_{2} .
$$

Step II. Let $\delta>0$. We will show that

$$
\left|u^{\varepsilon}-u\right|<2 \delta \text { in } \bar{Q}
$$

if $\varepsilon$ is small enough. 
From (6.3), it follows that if $\bar{l}>l_{1}$ is such that $\bar{k}_{1} e^{-\bar{k}_{2} \bar{l}^{2}} \leq 2 \delta$, then

$$
\left|u^{\varepsilon}-u\right|<2 \delta \text { in } x_{1}<-\bar{l} \text {, if } \varepsilon \leq \varepsilon_{1} .
$$

We now proceed as in the proof of Theorem 6.1. For $\rho>0$ and $\sigma>0$ small, we define in $\bar{Q}$

$$
u_{\rho, \sigma}(x, t)=(1+\sigma) u\left(x_{1}+\rho, x^{\prime}, t\right) .
$$

Since $\|u\|_{C^{\alpha, \frac{\alpha}{2}}(\bar{Q})}<\infty$, there exists $c>0$ such that

$$
\left|u_{\rho, \sigma}(x, t)-u(x, t)\right| \leq \sigma \mathcal{A}+c \rho^{\alpha} \quad \text { in } \bar{Q},
$$

where $\mathcal{A} \geq\|u\|_{L^{\infty}(Q)}$.

Let us fix $0<\rho<1$ such that $c \rho^{\alpha} \leq \frac{\delta}{2}$. We now fix $l \geq \max \left\{\bar{l}, l_{1}, l_{2}\right\}+1$, such that $\bar{k}_{1} e^{-\frac{\bar{k}_{2}}{2} l^{2}} \leq \frac{\bar{c}_{1}}{2} e^{-\bar{c}_{2} l}$ and such that $\rho \geq e^{-\frac{\bar{k}_{2}}{2} l^{2}}$.

We finally fix $\sigma>0$ such that $\sigma \mathcal{A} \leq \min \left\{\frac{\delta}{2}, \frac{\bar{c}_{1}}{4} e^{-\frac{\bar{k}_{2}}{2} l^{2}-\bar{c}_{2} l}\right\}$. Since $u_{x_{1}}<0$ in $\overline{\{u>0\}} \cap\{t=0\}, \sigma$ may be chosen small enough so that

$$
u_{\rho, \sigma}(x, 0)<u(x, 0) \quad \text { in } \overline{\left\{u_{\rho, \sigma}(x, 0)>0\right\}} \cap\left\{x_{1} \geq-l\right\} .
$$

We will next show that, if $\varepsilon$ is small enough,

$$
u_{\rho, \sigma}-\delta \leq u^{\varepsilon} \quad \text { in } \bar{Q} \cap\left\{x_{1} \geq-l\right\} .
$$

First, let us see that there holds, for $\varepsilon \leq \varepsilon_{1}$, that

$$
u_{\rho, \sigma}\left(-l, x^{\prime}, t\right) \leq u^{\varepsilon}\left(-l, x^{\prime}, t\right)-\delta(l) \text { for } x^{\prime} \in \bar{\Sigma}, 0 \leq t \leq T
$$

with $\delta(l)>0$. In fact, by (6.3) and (6.4)

$$
(1+\sigma) u\left(-l+\rho, x^{\prime}, t\right)-u^{\varepsilon}\left(-l, x^{\prime}, t\right) \leq-\bar{c}_{1} e^{\bar{c}_{2}(-l+\theta \rho)} \rho+\bar{k}_{1} e^{-\bar{k}_{2} l^{2}}+\sigma \mathcal{A}
$$

where $0<\theta<1$. Thus,

$$
\begin{aligned}
u_{\rho, \sigma}\left(-l, x^{\prime}, t\right)-u^{\varepsilon}\left(-l, x^{\prime}, t\right) & \leq e^{-\frac{\bar{k}_{2}}{2} l^{2}}\left(\bar{k}_{1} e^{-\frac{\bar{k}_{2}}{2} l^{2}}-\bar{c}_{1} e^{-\bar{c}_{2} l}\right)+\sigma \mathcal{A} \\
& \leq-\frac{\bar{c}_{1}}{2} e^{-\frac{\bar{k}_{2}}{2} l^{2}-\bar{c}_{2} l}+\sigma \mathcal{A} \leq-\frac{\bar{c}_{1}}{4} e^{-\frac{\bar{k}_{2}}{2} l^{2}-\bar{c}_{2} l}:=-\delta(l) .
\end{aligned}
$$

Since $u_{\rho, \sigma}$ satisfies the hypotheses of Theorem 5.1, we let $v^{\varepsilon}$ be a family of weak subsolutions to $P_{\varepsilon}$ in $Q$ converging to $u_{\rho, \sigma}$ uniformly in $\bar{Q}$, constructed as in Theorem 5.1. Then, by (6.8), if $\varepsilon$ is small enough,

$$
v^{\varepsilon} \leq u^{\varepsilon} \quad \text { on } x_{1}=-l .
$$

On the other hand, from (6.6), it follows that, for $\varepsilon$ small,

$$
v^{\varepsilon}(x, 0) \leq u^{\varepsilon}(x, 0) \quad \text { on } x_{1} \geq-l .
$$

We deduce that $v^{\varepsilon} \leq u^{\varepsilon}$ in $\bar{Q} \cap\left\{x_{1} \geq-l\right\}$, and therefore, (6.7) holds.

Since $\rho$ and $\sigma$ were chosen so that

$$
\left|u_{\rho, \sigma}(x, t)-u(x, t)\right| \leq \delta \quad \text { in } \bar{Q},
$$

we finally deduce from (6.7) that

$$
u-2 \delta \leq u^{\varepsilon} \quad \text { in } \bar{Q} \cap\left\{x_{1} \geq-l\right\}
$$

if $\varepsilon$ is small. 
Therefore, by (6.5) and (6.9) there holds that

$$
u-2 \delta \leq u^{\varepsilon} \quad \text { in } \bar{Q}
$$

if $\varepsilon$ is small enough.

We proceed analogously to deduce that

$$
u^{\varepsilon} \leq u+2 \delta \quad \text { in } \bar{Q}
$$

if $\varepsilon$ is small enough. The theorem is proved.

\section{APPENDIX}

In this Appendix we prove that a point $\left(x_{0}, t_{0}\right) \in \partial_{N} \mathcal{R} \subset \partial_{p} \mathcal{R}$ for the problem $\Delta v+\sum a_{i} v_{x_{i}}-v_{t}=\psi(v)$ in $\mathcal{R}, \frac{\partial v}{\partial \eta}=0$ on $\partial_{N} \mathcal{R}$, can be seen as an interior point for an equation with principal part in divergence form. In addition, if $\partial_{N} \mathcal{R}$ and $\partial_{D} \mathcal{R}=\partial_{p} \mathcal{R} \backslash \partial_{N} \mathcal{R}$ (on which Dirichlet data are given) are transversal, the new region has a Dirichlet boundary with the same regularity as the original one. The result is also true if $\left(x_{0}, t_{0}\right)$ lies in $\overline{\partial_{N} \mathcal{R}} \cap \partial_{D} \mathcal{R}$.

We will consider a domain $\mathcal{R}=\mathcal{O} \times\left(T_{1}, T_{2}\right) \cap\{F(x, t)>0\}$. Here $\mathcal{O}=B_{R}\left(x_{0}\right) \cap$ $\{G(x)>0\}$ where $G \in C^{3}\left(\overline{B_{R}\left(x_{0}\right)}\right)$ with $|\nabla G| \neq 0$ on $\{G=0\}, G\left(x_{0}\right)=0$ and $F \in C\left(\overline{\mathcal{O}} \times\left[T_{1}, T_{2}\right]\right)$.

We prove the following result.

Proposition A.1. Let $\psi \in L^{\infty}(\mathbb{R})$ be globally Lipschitz continuous and let $a_{i} \in$ $L^{\infty}\left(\mathbb{R}^{N+1}\right)$. Let $v \in C(\overline{\mathcal{R}})$, with $\nabla v \in L^{2}(\mathcal{R})$, be a weak solution to

$$
\begin{aligned}
& \Delta v+\sum a_{i} v_{x_{i}}-v_{t}=\psi(v) \quad \text { in } \mathcal{R}, \\
& \frac{\partial v}{\partial \eta}=0 \quad \text { on } \partial_{N} \mathcal{R},
\end{aligned}
$$

where $\partial_{N} \mathcal{R}=\left(B_{R}\left(x_{0}\right) \times\left(T_{1}, T_{2}\right)\right) \cap\{F>0\} \cap\{G=0\}$ and $\eta(x)=\frac{\nabla G(x)}{|\nabla G(x)|}$.

Then, there exist $0<r \leq R$, a neighborhood $\mathcal{N}$ of the origin in the variables $\left(y_{1}, \ldots, y_{N}\right)$ and an invertible function $h \in C^{2}\left(B_{r}\left(x_{0}\right), \mathcal{N}\right)$ with nonvanishing Jacobian in $B_{r}\left(x_{0}\right)$, which are independent of the function $F$, such that $h\left(x_{0}\right)=0$ and $h\left(\mathcal{O} \cap B_{r}\left(x_{0}\right)\right)=\mathcal{N} \cap\left\{y_{N}>0\right\}, \frac{\partial h^{-1}}{\partial y_{N}}(y)=\eta\left(h^{-1}(y)\right)$ on $\left\{y_{N}=0\right\}$, and such that the following result holds:

For $t \in\left[T_{1}, T_{2}\right]$, let

$$
\hat{F}(y, t)=F\left(h^{-1}(y), t\right), \quad y \in \mathcal{N} \cap\left\{y_{N}>0\right\},
$$

and

$$
\tilde{F}(y, t)=\left\{\begin{array}{l}
\hat{F}\left(y_{1}, \ldots, y_{N-1},-y_{N}, t\right), \quad y \in \mathcal{N} \cap\left\{y_{N} \leq 0\right\} \\
\hat{F}(y, t), \quad y \in \mathcal{N} \cap\left\{y_{N}>0\right\}
\end{array}\right.
$$

Let us define analogously the function $\tilde{v}(y, t)$. Then, there holds that $\tilde{v}$ is continuous in $\left(\mathcal{N} \times\left[T_{1}, T_{2}\right]\right) \cap\{\tilde{F} \geq 0\}$ with $\nabla \tilde{v} \in L^{2}\left(\left(\mathcal{N} \times\left(T_{1}, T_{2}\right)\right) \cap\{\tilde{F}>0\}\right)$ and $\tilde{v}$ is a weak solution of the following uniformly parabolic equation:

$$
\sum_{i, j} \frac{\partial}{\partial y_{i}}\left(a_{i j}(y) \frac{\partial}{\partial y_{j}} \tilde{v}(y, t)\right)+\sum_{i} b_{i}(y, t) \frac{\partial \tilde{v}}{\partial y_{i}}(y, t)-\tilde{v}_{t}=\psi(\tilde{v}(y, t)),
$$

in $\left(\mathcal{N} \times\left(T_{1}, T_{2}\right)\right) \cap\{\tilde{F}>0\}$, where $a_{i j} \in W_{l o c}^{1, \infty}(\mathcal{N}), b_{i} \in L_{\text {loc }}^{\infty}\left(\mathcal{N} \times\left[T_{1}, T_{2}\right]\right)$ are independent of the function $F$. 
Let $\nu \in \mathbb{R}^{N}$ be such that $|\nu|=1$ and $\nabla G\left(x_{0}\right) \cdot \nu=0$. We may choose the coordinates $\left(y_{1}, \ldots, y_{N}\right)$ in such a way that $\nabla h_{1}\left(x_{0}\right)=\nu, \nabla h_{i}\left(x_{0}\right) \cdot \nabla h_{j}\left(x_{0}\right)=\delta_{i j}$ and $a_{i j}(0)=\delta_{i j}$.

If $F \in C^{1}, \nabla F \in C^{\alpha, \frac{\alpha}{2}}$ in a neighborhood of $\{F=0\}$ up to $\{G=0\}, \nabla F(x, t)$. $\nabla G(x)=0$ for every $(x, t)$ in that neighborhood such that $G(x)=0$, and $|\nabla F| \geq$ $c>0$ on $\{F=0\}$, then there holds that $\tilde{F} \in C^{1}, \nabla \tilde{F} \in C^{\alpha, \frac{\alpha}{2}}$ in the corresponding neighborhood of $\{\tilde{F}=0\}$ and $\nabla \tilde{F} \neq 0$ on $\{\tilde{F}=0\}$.

Moreover, if $v\left(x, T_{1}\right) \in C^{1+\alpha}$ in a neighborhood of $\left\{F\left(x, T_{1}\right)=0\right\}$ up to $\{G=0\}$, $\nabla v\left(x, T_{1}\right) \cdot \nabla G(x)=0$ for every $x$ in that neighborhood such that $G(x)=0$, there holds that $\tilde{v}\left(y, T_{1}\right) \in C^{1+\alpha}$ in the corresponding neighborhood of $\left\{\tilde{F}\left(y, T_{1}\right)=0\right\}$.

Proof. Let $r>0$ be small so that there exists a regular $C^{3}$ parameterization $\sigma$ of $\{G=0\} \cap B_{r}\left(x_{0}\right)$ in the variables $y^{\prime}=\left(y_{1}, \ldots, y_{N-1}\right)$ in a neighborhood of the origin in $\mathbb{R}^{N-1}$. Let $\eta(x)$ be the interior unit normal to $\{G>0\}$ at $x \in\{G=0\}$. Then if $r$ is taken small enough, every point $x \in B_{r}\left(x_{0}\right)$ can be written in a unique way as

$$
x=\sigma\left(y^{\prime}\right)+y_{N} \eta\left(\sigma\left(y^{\prime}\right)\right):=h^{-1}(y)
$$

with $y \in \mathcal{N}$ a neighborhood of the origin in $\mathbb{R}^{N}$ and $h \in C^{2}\left(B_{r}\left(x_{0}\right), \mathcal{N}\right)$ with nonvanishing Jacobian in $B_{r}\left(x_{0}\right)$. Then $h\left(\mathcal{O} \cap B_{r}\left(x_{0}\right)\right)=\mathcal{N} \cap\left\{y_{N}>0\right\}$.

Let $\nu \in \mathbb{R}^{N}$ be as in the statement. We may choose the parameterization $\sigma$ in such a way that $\sigma(0)=x_{0}, \frac{\partial \sigma}{\partial y_{1}}(0)=\nu$ and $\left\{\frac{\partial \sigma}{\partial y_{i}}(0)\right\}_{1 \leq i \leq N-1}$ are orthonormal vectors.

Let $\tilde{v}$ and $\tilde{F}$ as in the statement. Then $\tilde{v}$ is a continuous function in $(\mathcal{N} \times$ $\left.\left[T_{1}, T_{2}\right]\right) \cap\{\tilde{F} \geq 0\}$. Since $v$ is a weak solution to (A.1), it is easy to see that $\tilde{v}$ is a weak solution to

$$
\left\{\begin{aligned}
& \sum_{i, j} \frac{\partial}{\partial y_{i}}\left(a_{i j}(y) \frac{\partial}{\partial y_{j}} \tilde{v}(y, t)\right)+\sum_{i} b_{i}(y, t) \frac{\partial \tilde{v}}{\partial y_{i}}(y, t)-\tilde{v}_{t}=\psi(\tilde{v}(y, t)) \text { in }\left(\mathcal{N} \times\left(T_{1}, T_{2}\right)\right) \cap\{\tilde{F}>0\} \cap\left\{y_{N}>0\right\} \\
& \sum_{i, j} a_{i j} \frac{\partial \tilde{v}}{\partial y_{j}} \eta_{i}=0 \quad \text { on }\left(\mathcal{N} \times\left(T_{1}, T_{2}\right)\right) \cap\{\tilde{F}>0\} \cap\left\{y_{N}=0\right\} .
\end{aligned}\right.
$$

Here $b_{i} \in L^{\infty}$ and $a_{i j}(y)=\nabla h_{i}(x) \cdot \nabla h_{j}(x), y=h(x)$. For $y_{N}<0$ let

$$
b_{i}(y, t)= \begin{cases}b_{i}\left(y^{\prime},-y_{N}, t\right) & \text { if } i<N \\ -b_{i}\left(y^{\prime},-y_{N}, t\right) & \text { if } i=N\end{cases}
$$

and

$$
a_{i j}(y)= \begin{cases}a_{i j}\left(y^{\prime},-y_{N}\right) & \text { if } i<N \text { and } j<N \text { or } i=j=N \\ -a_{i j}\left(y^{\prime},-y_{N}\right) & \text { if } i=N \text { and } j<N \text { or } i<N \text { and } j=N\end{cases}
$$

Then $\tilde{v}$ is a weak solution to $(\mathrm{A} .2)$ in $\left(\mathcal{N} \times\left(T_{1}, T_{2}\right)\right) \cap\{\tilde{F}>0\}$.

Let us see that

$$
\sum_{i, j} a_{i j}(y) \xi_{i} \xi_{j} \geq \lambda|\xi|^{2}
$$


for some $\lambda>0$. In fact, for $y_{N} \geq 0$

$$
\sum_{i, j} a_{i j}(y) \xi_{i} \xi_{j}=\sum_{i, j} \nabla h_{i}(x) \xi_{i} \cdot \nabla h_{j}(x) \xi_{j}=\left|(D h(x))^{T} \xi\right|^{2} \geq \lambda|\xi|^{2} .
$$

From the fact that (A.3) holds for $y_{N} \geq 0$ and from the definition of $a_{i j}$ we see that (A.3) holds in $\left\{y_{N}<0\right\}$.

Clearly, $b_{i} \in L_{\text {loc }}^{\infty}\left(\mathcal{N} \times\left[T_{1}, T_{2}\right]\right)$. Let us see that $a_{i j} \in W_{\text {loc }}^{1, \infty}(\mathcal{N})$. On one hand, $a_{i j} \in C^{1}\left(\mathcal{N} \cap\left\{y_{N} \geq 0\right\}\right) \cap C^{1}\left(\mathcal{N} \cap\left\{y_{N} \leq 0\right\}\right)$. On the other hand, $a_{i j} \in C(\mathcal{N})$ since $a_{i N}\left(y^{\prime}, 0\right)=0$ for $i<N$. Indeed, from the fact that $h\left(\sigma\left(y^{\prime}\right)+y_{N} \eta\left(\sigma\left(y^{\prime}\right)\right)\right)=y$ it follows that, on $\{G=0\}, \nabla h_{i}$ is tangent to $\{G=0\}$ if $i<N$ and $\nabla h_{N}=\eta$. Therefore,

$$
\nabla h_{i}(x) \cdot \nabla h_{N}(x)=0 \quad \text { for } x \in\{G=0\} \quad \text { if } i<N .
$$

In addition, it can be seen that $\nabla h_{i}\left(x_{0}\right)=\frac{\partial \sigma}{\partial y_{i}}(0)$ for $i<N$. Thus, $a_{i j}(0)=\delta_{i j}$ and $\nabla h_{1}\left(x_{0}\right)=\nu$.

When $F$ is smooth in a neighborhood of $\{F=0\}$, we use the fact that $\nabla F(x, t)$. $\nabla G(x)=0$ on $\{G=0\}$ implies that $\frac{\partial \tilde{F}}{\partial y_{N}}=0$ on $\left\{y_{N}=0\right\}$ in order to prove that $\tilde{F}$ is smooth.

We proceed analogously with $v\left(x, T_{1}\right)$.

Remark A.1. In Proposition A.1 we may consider sub- or supersolutions instead of solutions and a similar conclusion holds.

\section{ACKNOWLEDGMENT}

Most of this work was performed while the second author was a visiting professor at the University of Buenos Aires (UBA). He wants to express his gratitude for their warm hospitality.

\section{REFERENCES}

[AG] D. Andreucci, R. Gianni, Classical solutions to a multidimensional free boundary problem arising in combustion theory, Comm. Partial Diff. Eq. 19 (1994), 803-826. MR 95h:35248

[BCN] H. Berestycki, L.A. Caffarelli, and L. Nirenberg, Uniform estimates for regularization of free boundary problems, "Analysis and Partial Differential Equations" (Cora Sadosky, ed.), Lecture Notes in Pure and Applied Mathematics, vol. 122, Marcel Dekker, New York, 1990, pp. 567-619. MR 91h:35112

[BL] H. Berestycki and B. Larrouturou, Quelques aspects mathématiques de la propagation des flammes prémélangées, Nonlinear Partial Differential Equations and their Applications, Collège de France Seminar (Brezis \& Lions, eds.), vol. 10, Pitman-Longman, Harlow, UK, 1991. MR 93a: 80008

[BNS] H. Berestycki, B. Nicolaenko, and B. Scheurer, Traveling wave solutions to combustion models and their singular limits, SIAM J. Math. Anal. 16 (1985), 1207-1242. MR 87h:35326

[BoG] A. Bonnet and L. Glangetas, Non-uniqueness for traveling fronts in the limit of high activation energy, preprint.

[BuL] J.D. Buckmaster and G.S.S. Ludford, Theory of Laminar Flames, Cambridge University Press, Cambridge, 1982. MR 84f:80011

[C1] L.A. Caffarelli, A monotonicity formula for heat functions in disjoint domains, "Boundary value problems for P.D.E.'s and applications", dedicated to E. Magenes (J.L. Lions, C. Baiocchi, eds.), Masson, Paris, 1993, pp. 53-60. MR 95e:35096

[C2] Uniform Lipschitz regularity of a singular perturbation problem, Differ. Integ. Equat. 8 (7) (1995), 1585-1590. MR 96i:35135 
[CK] L.A. Caffarelli and C. Kenig, Gradient estimates for variable coefficient parabolic equations and singular perturbation problems, Amer. J. Math. 120 (2) (1998), 391-439. MR 99b:35081

[CLW1] L.A. Caffarelli, C. Lederman, and N. Wolanski, Uniform estimates and limits for a two phase parabolic singular perturbation problem, Indiana Univ. Math. J. 46 (2) (1997), 453-490. MR 98i:35099

[CLW2] L.A. Caffarelli, C. Lederman, and N. Wolanski, Pointwise and viscosity solutions for the limit of a two phase parabolic singular perturbation problem, Indiana Univ. Math. J. 46 (3) (1997), 719-740. MR 99c:35116

[CV] L.A. Caffarelli and J.L. Vazquez, A free boundary problem for the heat equation arising in flame propagation, Trans. Amer. Math. Soc. 347 (1995), 411-441. MR 95e:35097

[GHV] V.A. Galaktionov, J. Hulshof, and J.L. Vazquez, Extinction and focusing behaviour of spherical and annular flames described by a free boundary problem, Jour. Math. Pure Appl. 76 (1997), 563-608. MR 98h:35328

[Gl] L. Glangetas, Etude d'une limite singulière d'un modèle intervenant en combustion, Asymptotic Analysis 5 (1992), 317-342. MR 93g:80010

[H] D. Henry, Geometric Theory of Semilinear Parabolic Equations, Lecture Notes in Mathematics, vol. 840, Springer-Verlag, New York, Berlin, 1981. MR 83j:35084

$[\mathrm{HH}]$ D. Hilhorst and J. Hulshof, An elliptic-parabolic problem in combustion theory: convergence to travelling waves, Nonlinear Anal. 17 (1991), 519-546. MR 92g:35242

$[\mathrm{KH}] \quad$ L.I. Kamynin and B.N. Himcenko, On applications of the maximum principle to parabolic equations of second order, Soviet Math. Doklady 13(3) (1972), 683-686.

[LSU] O.A. Ladyzenskaja, V.A. Solonnikov, and N.N. Uralceva, Linear and Quasilinear Equations of Parabolic Type, Translations of Mathematical Monographs, vol. 23, AMS, Providence, Rhode Island, USA, 1967. MR 39:3159b

[LVW] C. Lederman, J.L. Vazquez, and N. Wolanski, A mixed semilinear parabolic problem in a noncylindrical space-time domain, Diff. Int. Eqs. (to appear).

[LW] C. Lederman and N. Wolanski, Viscosity solutions and regularity of the free boundary for the limit of an elliptic two phase singular perturbation problem, Annali Scuola Norm. Sup. Pisa Cl. Sci. (4) 27 (1998), 253-288. [MR 99m:35274]

[M] A.M. Meirmanov, On a free boundary problem for parabolic equations, Matem. Sbornik 115 (1981), 532-543 (in Russian); English translation: Math. USSR Sbornik, 43 (1982), $473-484$.

[V] J.L. Vazquez, The free boundary problem for the heat equation with fixed gradient condition, Free Boundary Problems, Theory and Applications, vol. 363, Longman, M. Niezgodka, P. Strzelecki eds., Pitman Research Series in Mathematics, 1996, pp. 277-302. MR 98h:35246

[Ve] Ventsel', A free boundary-value problem for the heat equation, Dokl. Akad. Nauk SSSR 131 (1960), 1000-1003 English translation: Soviet Math. Dokl., 1 (1960).

[W] F.A. Williams, Combustion Theory, 2nd. ed., Benjamin-Cummnings, Menlo Park, CA, 1985.

[ZF] Ya.B. Zeldovich and D.A. Frank-Kamenetski, The theory of thermal propagation of flames, Zh. Fiz. Khim. 12 (1938), 100-105 (in Russian); English translation in "Collected Works of Ya.B. Zeldovich", vol. 1, Princeton Univ. Press, 1992.

Departamento de Matemática, Facultad de Ciencias Exactas y Naturales, Universidad de Buenos Aires, (1428), Buenos Aires, Argentina

E-mail address: clederma@dm.uba.ar

Departamento de Matemáticas, Universidad Autónoma de Madrid, 28049 Madrid, SPAIN

E-mail address: juanluis.vazquez@uam.es

Departamento de Matemática, Facultad de Ciencias Exactas y Naturales, Universidad de Buenos Aires, (1428), Buenos Aires, Argentina

E-mail address: wolanski@dm.uba.ar 